\title{
The Effect of Cumulus Cloud Field Anisotropy on Domain-Averaged Solar Fluxes and Atmospheric Heating Rates
}

\author{
LAURA M. HiNKELMAN \\ National Institute of Aerospace, Hampton, Virginia \\ K. FrankLin Evans \\ Department of Atmospheric and Oceanic Sciences, University of Colorado, Boulder, Colorado \\ Eugene E. Clothiaux \\ Department of Meteorology, The Pennsylvania State University, University Park, Pennsylvania \\ Thomas P. ACKERMAN \\ Fundamental Sciences Division, Pacific Northwest National Laboratory, Richland, Washington \\ Paul W. Stackhouse JR. \\ NASA Langley Research Center, Hampton, Virginia
}

(Manuscript received 1 September 2006, in final form 11 January 2007)

\begin{abstract}
Cumulus clouds can become tilted or elongated in the presence of wind shear. Nevertheless, most studies of the interaction of cumulus clouds and radiation have assumed these clouds to be isotropic. This paper describes an investigation of the effect of fair-weather cumulus cloud field anisotropy on domain-averaged solar fluxes and atmospheric heating rate profiles. A stochastic field generation algorithm was used to produce 20 three-dimensional liquid water content fields based on the statistical properties of cloud scenes from a large eddy simulation. Progressively greater degrees of $x-z$ plane tilting and horizontal stretching were imposed on each of these scenes, so that an ensemble of scenes was produced for each level of distortion. The resulting scenes were used as input to a three-dimensional Monte Carlo radiative transfer model. Domain-averaged transmission, reflection, and absorption of broadband solar radiation were computed for each scene along with the average heating rate profile. Both tilt and horizontal stretching were found to significantly affect calculated fluxes, with the amount and sign of flux differences depending strongly on sun position relative to cloud distortion geometry. The mechanisms by which anisotropy interacts with solar fluxes were investigated by comparisons to independent pixel approximation and tilted independent pixel approximation computations for the same scenes. Cumulus anisotropy was found to most strongly impact solar radiative transfer by changing the effective cloud fraction (i.e., the cloud fraction with respect to the solar beam direction).
\end{abstract}

\section{Introduction}

The effect of three-dimensional (3D) cloud variability on the transport of solar flux in the atmosphere has been an active area of research for the last three de-

Corresponding author address: Dr. Laura Hinkelman, National Institute of Aerospace, 100 Exploration Way, Rm. 214, Hampton, VA 23666.

E-mail: 1.m.hinkelman@larc.nasa.gov cades (e.g., McKee and Cox 1974; Aida 1977; Davies 1978; Harshvardhan and Thomas 1984; Welch and Wielicki 1984; Barker and Davies 1992; Cahalan et al. 1994b; Marshak et al. 1995b; Barker et al. 1998; O'Hirok and Gautier 1998; Zuidema and Evans 1998; Benner and Evans 2001; Scheirer and Macke 2003; Di Giuseppe and Tompkins 2005). The 3D radiative transfer effect on domain-averaged solar fluxes has been divided into two physical processes, as summarized by Várnai and Davies (1999). The first, which is termed 
the "one-dimensional (1D) heterogeneity effect," arises from the nonlinear relationship between cloud optical depth and albedo. The mean transmission of a cloud with horizontally varying optical depth is more than the transmission of a uniform cloud with the mean optical depth. As a result, computed fluxes will be in error if a region with horizontally varying cloud properties is treated as homogeneous. The second process, which may be termed the "horizontal transport effect," involves the horizontal propagation of radiation. Photons striking the top of a cloud can leak out the sides or diffuse out the top. For low solar elevations, photons can illuminate the side of a cloud, shading other parts of the field. Conversely, photons exiting cloud sides can enter the sides of other clouds and be scattered out of their tops. All of these processes can affect a cloud field's domain-averaged radiative properties, such as reflectance and transmittance.

The independent pixel (or column) approximation (IPA; Ronnholm et al. 1980; Cahalan 1989) was devised to treat cloud horizontal heterogeneity by integrating 1D (plane parallel) radiative transfer over the distribution of optical depths in a scene. The IPA has been found to be an accurate approximation for the computation of solar fluxes in overcast stratiform clouds (Cahalan et al. 1994b; Chambers et al. 1997; Zuidema and Evans 1998). However, even if 1D heterogeneity is accounted for, the horizontal transport effect causes potentially large solar flux errors in cumulus cloud fields (Barker 1996; Chambers et al. 1997; Benner and Evans 2001). The horizontal transport effect can be divided into two components. One component results from the way the projected cloud properties change as a function of solar zenith angle. For example, the effective cloud fraction (Harshvardhan and Thomas 1984) of cumulus clouds tends to increase with solar zenith angle because the sun illuminates the sides and tops of the clouds. The tilted independent pixel approximation (TIPA; Várnai and Davies 1999) attempts to account for this effect by performing radiative transfer on independent columns oriented along the slant path of incoming solar photons. This is equivalent to applying the IPA to a distribution of cloud optical depths adjusted for the angle of solar incidence. The second component of the horizontal transport effect is photon transport perpendicular to the incident solar direction. This component includes radiative smoothing (Marshak et al. 1995a) and cloud side leakage (Várnai and Davies 1999). Perpendicular photon transport can only be correctly computed using full 3D radiative transfer techniques and thus requires knowledge of the complete structure of cloud fields.

An ongoing problem in the field of 3D radiative transfer in the atmosphere has been the lack of $3 \mathrm{D}$ cloud information. Most measurements are limited to the horizontal plane (e.g., satellite) or vertical cross sections (e.g., radar). The lack of 3D cloud structure data has limited our ability to accurately assess the realworld importance of $3 \mathrm{D}$ radiative transport. It has also limited the types of cloud structures that have been investigated. One aspect of cloud geometry that has largely been ignored is anisotropy (i.e., structural variability that depends on the sampling direction). This oversight requires attention because anisotropy is common in broken cloud fields. Individual clouds may be elongated in a horizontal direction or tilted out of the vertical. On the larger scale, cloud fields frequently exhibit preferred directions, the most extreme example being cloud streets.

Barker and Davies (1992) briefly addressed the importance of horizontal anisotropy in broken cloud fields to radiative transfer in climate models. Using a test cloud field based on a strongly anisotropic Advanced Very High Resolution Radiometer (AVHRR) scene, they found that reflectance varied by as much as $5 \%$ as a function of solar azimuth angle. They also detected smaller differences between azimuthally averaged reflectances computed for the anisotropic field and the corresponding reflectances for statistically similar isotropic scenes. They concluded that isotropic clouds may be a sufficient approximation for anisotropic fields in climate models. This study had two weaknesses. First, only one anisotropic scene was examined. Second, the cloud fields on which these tests were run were essentially two dimensional (only a single pixel thick) and had a uniform optical depth, $\tau$. Since more recent studies (Cahalan et al. 1994a; Titov 1998; O'Hirok and Gautier 1998; Zuidema and Evans 1998; Várnai and Davies 1999) have shown that both horizontal $\tau$ variations and vertical cloud structure are important to radiative transport in the cloudy atmosphere, further investigation of the radiative impact of horizontal anisotropy is required.

Barker (1994) also examined the effect of shearinduced tilt on the albedo of cumulus clouds. This study was based on regular arrays of cuboidal clouds having a uniform volume extinction coefficient. The clouds were tilted to selected angles by consecutively offsetting the vertical layers in the horizontal direction. The albedos of the resulting cloud fields differed from the original field albedos by as much as $25 \%$. These differences depended strongly on solar azimuthal angle as well as cloud tilt and were attributed primarily to changes in the effective cloud fraction. Since integration over all azimuthal angles reduced the total albedo difference to $5 \%-10 \%$, it was speculated that sheared cloud structures may not be significant in a climatological sense. 
This study relied primarily on model clouds with idealized geometric shapes and did not explore the effects of tilt on atmospheric heating rate profiles, leaving many questions unanswered.

The current study attempts to improve our understanding of the impact of cumulus cloud field anisotropy on solar radiative transfer and to elucidate the mechanisms through which these effects occur. Our ultimate purpose is to determine when, or whether, cloud field anisotropy must be accounted for. The input to this study is a set of stochastically generated cloud fields upon which varying degrees of anisotropy have been imposed. A large number of fully 3D fair-weather continental cumulus scenes are considered to give statistical weight to the results.

\section{Method}

\section{a. Background}

\section{1) Anisotropy measures}

In this paper, we consider two orthogonal types of anisotropy. The first, which we term horizontal anisotropy, corresponds to the "out of roundness" or elongation of cloud field structures in a horizontal plane. The second is tilt, or the degree to which the cloud structure shifts horizontally as a function of height. Separate measures are used to quantify these types of distortion. Since the methods used to compute these measures were presented in an earlier paper (Hinkelman et al. 2005), we limit our discussion to the interpretation of these two variables.

The anisotropy parameter (AP) is used to describe the degree to which the structures in a two-dimensional field vary as a function of direction. An individual cloud element is horizontally isotropic if its cross section in the horizontal plane is circular. It is anisotropic if the cross section is elliptical or linear. Anisotropy can also occur due to the arrangement of multiple cloud elements. For example, the elements could be spread randomly (an isotropic arrangement), linearly (highly anisotropic), or more closely packed in one direction than the other (somewhat anisotropic). The anisotropy parameter is sensitive to both of these sources of directionality.

The anisotropy parameter consists of an amplitude and an angle. It can be calculated as a function of spatial scale or include a range of scales. In this work, it is the average of the anisotropy values for scales from 140 to $1130 \mathrm{~m}$ [i.e., the smallest resolvable scale in the large eddy simulation (LES) model to roughly $20 \%$ of the domain size]. The significance of an AP amplitude depends on the number of elements in the $2 \mathrm{D}$ field and can be evaluated using a statistical significance test as described in Hinkelman et al. (2005). The AP amplitudes range from 0 for an isotropic field to 1 for a field that is perfectly linear. The AP angle indicates the direction of the long axis of the field's structure. We specify AP angles according to meteorological conventions [i.e., $0^{\circ}$ or $360^{\circ}$ denotes a north-south (vertical) axis of anisotropy while $90^{\circ}$ or $270^{\circ}$ refers to an eastwest (horizontal) orientation]. (Note that anisotropy angles are unique only over a range of $180^{\circ}$, since they decribe alignment parallel to an axis rather than pointing in a single direction.)

Vertical tilt is defined as the horizontal shift of cloud field structure per unit height. This value is computed for every pair of neighboring horizontal slices through the cloud field, then averaged over the entire depth of the field. Tilt is expressed as a unitless quantity (in $\mathrm{m} \mathrm{m}^{-1}$ ) or in degrees away from the vertical.

\section{2) Choice of SCAle}

In this study, we have chosen to focus on clouds represented at a resolution of 10-100 m. This is the limit to which cloud structure can be routinely measured at this time. Radar and lidar instruments, which provide some of the most detailed continuous measurements of cloud structure, are currently limited to vertical resolutions on the order of $10 \mathrm{~m}$. Solar radiation also interacts with clouds on scales of tens of meters. For these reasons we employed cloud fields with a domain size of $6.4 \mathrm{~km}$ and $66.67-\mathrm{m}$ sampling in the radiative transfer computations presented here.

\section{b. Stochastic cloud fields}

To isolate the effects of cumulus anisotropy on solar radiative transfer, we required a set of cloud fields for which the horizontal and vertical anisotropy varied individually and systematically while other characteristics important to radiative transport remained fixed. Although a number of LES liquid water content (LWC) fields were available from our previous study of anisotropy in fair-weather cumuli over Oklahoma (Hinkelman et al. 2005), tilt and horizontal anisotropy varied randomly and simultaneously among these scenes. At the same time, cloud fraction, total liquid water, and cloud structure also fluctuated. Rather than using such irregular test cases, we chose to study clouds produced by a modified version of the stochastic field generation algorithm of Evans and Wiscombe (2004) in which anisotropy values could be prescribed. Multiple output cloud fields with matching LWC distributions and correlation structure were produced for each set of anisotropy values. Averaging the results of the radiative 
transfer computations over these ensembles of similar fields allowed us to minimize the impact of variations in cloud structure in individual scenes.

The cloud field generation technique of Evans and Wiscombe (2004) is an extension of earlier methods of producing random two-dimensional fields with a known power spectrum under the assumptions of translational invariance and isotropy (e.g., Barker and Davies 1992; Evans 1993). The basic technique consists of filtering, or multiplying point-by-point in Fourier space, Gaussian random deviates by the desired amplitude spectrum and then Fourier transforming the output back to real space. The one-point statistics of the resulting fields remain Gaussian. If desired, the output array can be exponentiated or thresholded to produce a broken cloud field, but this changes the overall power spectrum.

Extending this technique to include the vertical dimension is nontrivial because the assumptions of isotropy and translational invariance do not hold in this direction (i.e., cloud structure does depend on height). Producing the desired vertical structure requires accounting for the correlation of LWC values at adjacent levels. Evans and Wiscombe (2004) generalized the Fourier filtering technique to the third dimension by using empirical orthogonal functions (EOFs) to decompose the vertical structure into independent (orthogonal) basis functions. (Note: Fourier series are the EOFs for translationally invariant fields.)

The Evans and Wiscombe (2004) technique is illustrated via a flowchart in their Fig. 2 and can be summarized as follows. First, the average cross-correlation function is computed for all possible level pairs from the binary cloud masks of all of the input scenes. (Cloud masks are chosen for the spatial structure statistics because the $3 \mathrm{D}$ radiative effects in cumulus clouds are believed to be mostly a function of cloud morphology.) The resulting "binary correlation matrix" as a function of horizontal distance is then converted to an equivalent "Gaussian correlation matrix." The frequency domain representation of the Gaussian correlation matrix is obtained via Fourier transform, and then an EOF decomposition is performed on each Fourier term (i.e., spatial frequency). This orthogonal function representation of the original correlation matrix is used as the basis of the stochastic cloud scenes.

To generate a new output field from this information, a field of Gaussian random variables with the same dimensions as the input correlation matrix is first generated. The random field is then multiplied point-bypoint by the filtering amplitudes, which are the square roots of the eigenvalues from the EOF decomposition.
The field is then transformed back into real space via consecutive EOF and Fourier transforms. Finally, the individual Gaussian values are mapped into the original LWC probability density function of the input scenes to obtain a broken cloud field. This process can be repeated using additional sets of Gaussian random deviates to produce any number of cloud scenes that are statistically similar to the population of cloud fields from which the horizontal power spectra and their vertical correlation properties were derived.

The reason that an equivalent Gaussian correlation matrix is used as the starting point of the field generation algorithm in place of the binary cloud mask correlation matrix is that the final mapping of stochastic Gaussian values to the original LWC distribution is nonlinear. This alters the power spectra of the output fields. The equivalent Gaussian correlation matrix is selected so that the average binary cloud mask correlation matrix of the output fields will approximately match the original binary mask correlation matrix in spite of the nonlinear transformation of the Gaussian output fields to LWC values.

Several modifications were made to the EvansWiscombe algorithm for this study. First, the use of input statistics from 3D LES LWC fields instead of 2D slices from upward-looking radar measurements was accommodated. This was accomplished by computing the mean 3D binary correlation matrix for the LES fields and then reducing this array to two dimensions by integrating the horizontal dimension azimuthally. The correlation matrix created in this way has the same form as correlation matrices from $2 \mathrm{D}$ radar slices. However, since the original correlation matrix is computed in three dimensions, it is no longer necessary to convert the power spectra from 2D fields to appropriate spectra for $3 \mathrm{D}$ fields as described in appendix 1 of Evans and Wiscombe (2004). In addition, any anisotropy that may have existed in the input fields is eliminated when the third dimension is lost. We are then able to incorporate horizontal anisotropy and tilt into the output fields in a controlled manner.

Horizontal anisotropy was added to stochastic cloud fields by elongating the LWC power spectrum for each vertical level in the north-south direction and compressing it in the east-west direction. This was done at the beginning of the cloud generation process, when the EOF vectors were multiplied by the Gaussian random deviates in the two-dimensional array. At every point in the array, $x, y$, instead of multiplying by the EOF vector corresponding to radial spatial frequency bin

$$
k_{r}=\sqrt{k_{x}^{2}+k_{y}^{2}}
$$


where $k_{x}$ and $k_{y}$ are the bin indices corresponding to positions $x$ and $y$, the EOF vector for spatial frequency bin $k_{r}^{\prime}$ was used. Here

$$
k_{r}^{\prime}=\sqrt{\left(k_{x} \text { stretch }\right)^{2}+\left(\frac{k_{y}}{\text { stretch }}\right)^{2}}
$$

and stretch is the parameter used to control the horizontal anisotropy. This produces a cloud field elongated in the east-west direction after the matrix is inverse Fourier transformed back to the spatial domain.

Controlling the degree of horizontal anisotropy produced in the output fields is complicated by the fact that conversion from the Gaussian distribution back to the original LWC values is a nonlinear operation. As a result, the final output clouds will not have the same degree of horizontal anisotropy as was set by the input parameters. Repeated trials are thus required to determine the parameter values required to obtain the desired levels of anisotropy.

Arbitrary vertical tilt from west to east was added by multiplying the Fourier amplitudes by a linear phase shift given by

$$
d \theta=e^{\left(k_{x} / c_{x} d x\right) \pi i},
$$

where $c_{x}$ is one-half the total number of fast Fourier transform (FFT) frequencies, $k_{x}$ is the horizontal (westeast) spatial frequency index, and $d x=i z$ tilt, where $i z$ is the index of the vertical cloud level and tilt is a parameter specified in horizontal grid spacings per vertical grid cell.

Two 3D LWC fields from each of six runs of the LES base case in Hinkelman et al. (2005) were used as input to the stochastic cloud field generation model. The six runs differed only in the random potential temperature fields used to initiate the convection. These welldeveloped fair-weather cumulus fields were all taken from simulation times between 8 and $9 \mathrm{~h}$ to provide a statistically similar ensemble of scenes. Vertical levels that were free of liquid water in all of the scenes were not included in the input to the stochastic cloud field program. For this reason, the output fields consisted of $96 \times 96 \times 37$ arrays at the LES grid spacing of $66.66 \times$ $66.66 \times 40.0 \mathrm{~m}^{3}$. Examples of LES scenes used as input to the algorithm are shown in Fig. 1.

Twenty realizations of isotropic cumulus cloud fields were generated using the stochastic method described above. Ten variations of each of these basic scenes were also produced by incorporating tilt or anisotropy. Five of the variations included tilts of $0.33,0.66,1.00,1.33$, and $1.66 \mathrm{~m} \mathrm{~m}^{-1}$ (corresponding to $0^{\circ}, 18^{\circ}, 33^{\circ}, 45^{\circ}, 53^{\circ}$, and $59^{\circ}$ from the vertical), while the other five had anisotropy parameters of approximately $0.15,0.25,0.35$,
0.45 , and 0.55 over the middle spatial frequencies. Note that these values were computed for individual scenes rather than as averages over 10 scenes as in Hinkelman et al. (2005). The anisotropy values used here were selected to cover a range from $0 \%$ to $\sim 160 \%$ of the maximum single scene value in the data from Hinkelman et al. (2005). Scenes with both tilt and horizontal anisotropy were not created so that it would be possible to isolate the effects of these two types of distortion. The degree to which we were able to control the anisotropy of the stochastic cloud fields is demonstrated in the graphs in Fig. 2.

A sample sequence of stochastic cloud fields with increasing levels of imposed horizontal anisotropy is shown in Fig. 3. The corresponding anisotropy parameter is indicated above each field. The anisotropy parameter value of 0.08 indicates the ensemble of nominally isotropic fields. This value is nonzero because some anisotropy occurs by chance in natural-looking cloud fields. Prior LES studies indicate that anisotropy parameter values of cloud fields range up to about 0.4 under typical meteorological conditions. Higher anisotropy parameters would occur only in extremely oriented fields, such as when cloud streets are present. The sequence of tilted clouds made using the same random components as the anisotropic fields in Fig. 3 is shown in Fig. 4. Note that imposing tilt in the west-east direction leaves the north-south direction unchanged. Mean (standard deviation) cloud properties for the isotropic scenes are cloud fractions of $0.24(0.05)$ and cloud optical depths of 19.2 (5.0). All of the clouds are less than $1450 \mathrm{~m}$ thick.

A comparison of the average cloud fraction as a function of height for the ensembles of stochastic fields with differing degrees of tilt and anisotropy is presented in the upper portion of Fig. 5. The ensemble-averaged cloud fraction varies so little as anisotropy increases that all of the mean value curves are coincident. At the cloudiest heights, the standard deviations of cloud fraction are higher for the stochastic ensembles than for the original group of scenes. However, the fact that the standard deviation curves are identical for all of the sets of anisotropic scenes suggests that this increase is a result of the limited number of stochastic scenes employed. Similar behavior is seen in the statistics of average LWC per cloud layer shown in the lower portion of Fig. 5. The average LWC at each height is also conserved as the tilt or horizontal anisotropy increases. The fact that the bulk cloud properties are so well conserved means that we will be able to make meaningful comparisons of the radiative fluxes and heating rates calculated for the fields as tilt and stretch are varied. 


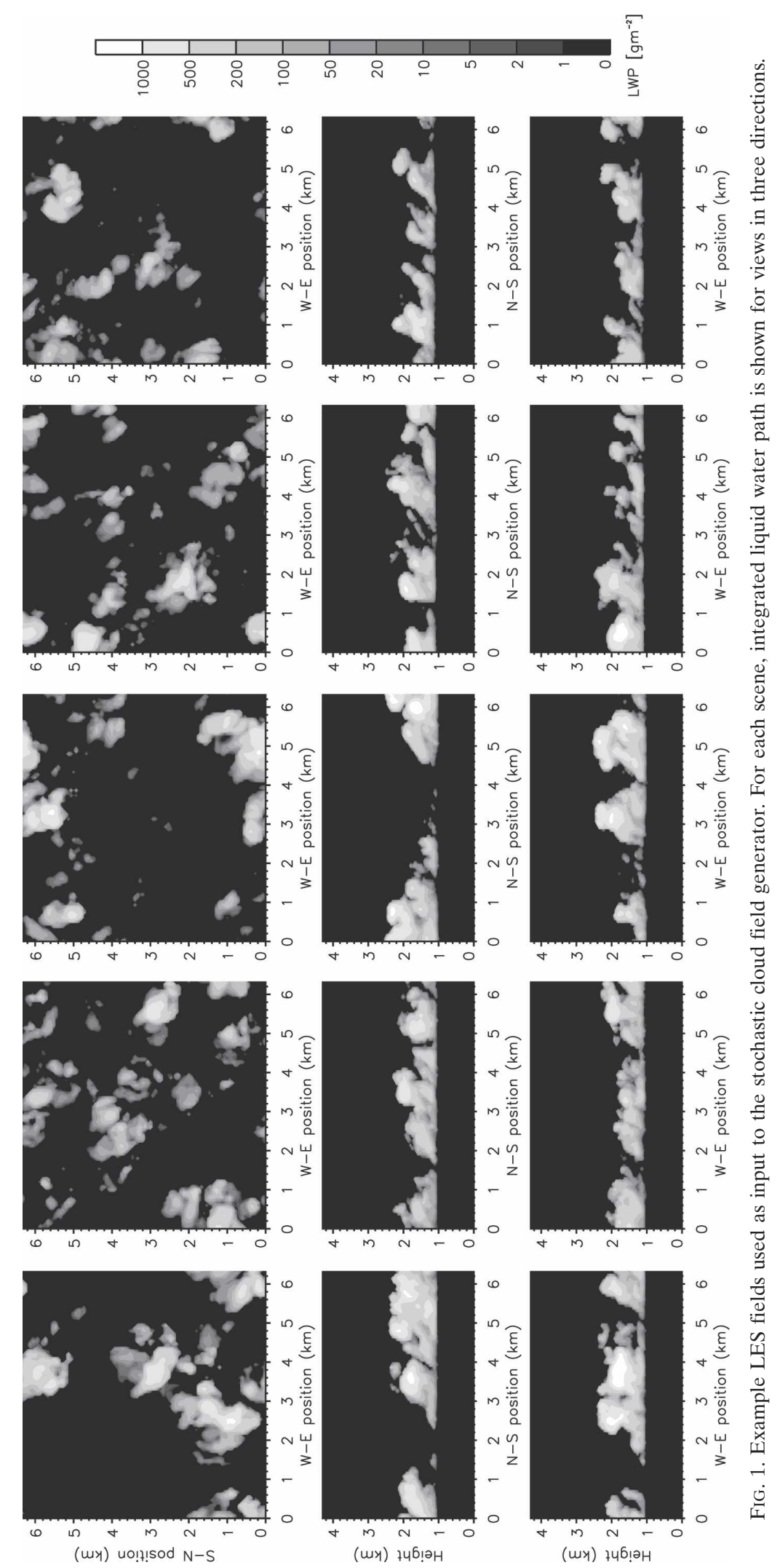



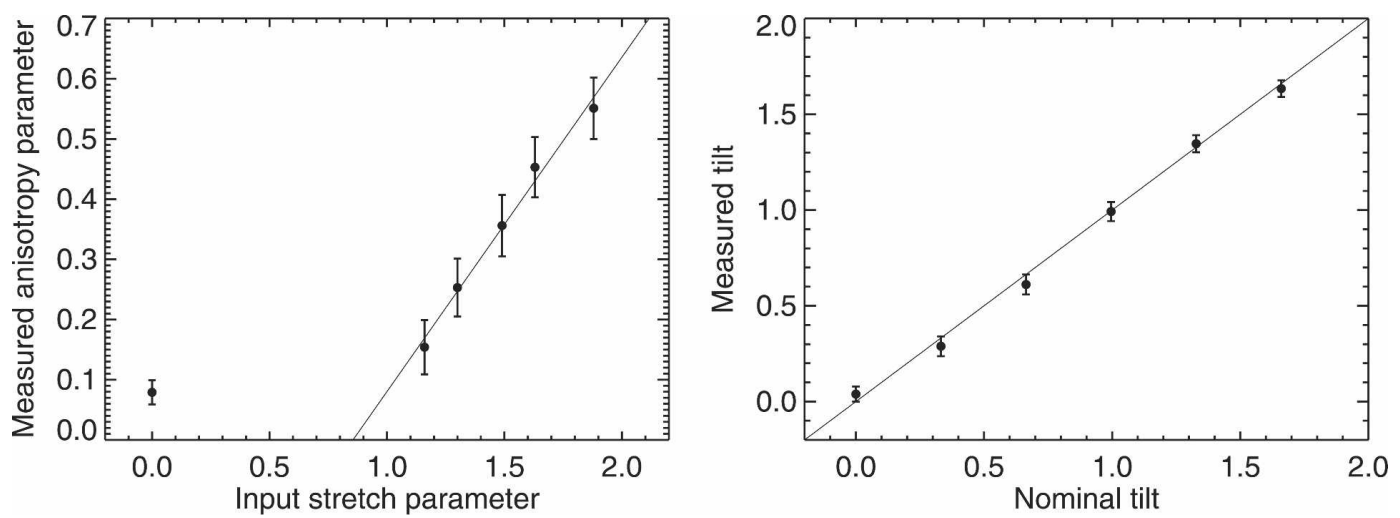

FIG. 2. (left) Horizontal anisotropy and (right) vertical tilt in stochastic cloud scenes as a function of the corresponding control parameter. Error bars indicate the standard error of the mean over the 20 -scene ensembles.

\section{c. Radiative transfer computations}

Monte Carlo radiative transfer calculations were performed for the 20 sequences of tilted or stretched cumulus scenes described above. The atmospheric property arrays required for the radiative transfer computations were created by inserting the LWC arrays from the stochastic cloud model into larger arrays defining the computational domain. The domain extended from the surface to $40.0 \mathrm{~km}$ with the cloudy layers between 1060 and $2500 \mathrm{~m}$. The cloud droplet effective radius in each cell was calculated from the local LWC value assuming a constant drop number density of $300 \mathrm{~cm}^{-3}$ and a lognormal droplet distribution with a logarithmic width of 0.38 (Miles et al. 2000). A single atmospheric profile of temperature, pressure, and water vapor density was used throughout the entire domain. Average values from the base LES cases were applied up to about $4000 \mathrm{~m}$. Above $4300 \mathrm{~m}$, the McClatchey midlatitude summer atmospheric profile was used. In between, the values were interpolated to splice the two curves together smoothly.

Solar radiative transfer through the cloud scenes was computed using a Monte Carlo model (Benner and Evans 2001) based on the maximal cross-section method of Marchuk (Marchuk et al. 1980) and tested by comparison to other algorithms in the Intercomparison of Radiation Codes in Climate Models Phase III (ICRCCM-III; Barker et al. 2003). The Monte Carlo algorithm included molecular Rayleigh scattering and incorporated molecular absorption via the shortwave rapid radiative transfer model (RRTM) correlated- $k$ distribution (Mlawer et al. 1997), comprising 14 bands from 0.2 to $12.2 \mu \mathrm{m}$. Optical properties averaged over each wavelength band were calculated explicitly using Mie theory for the droplet distributions. Up to 2000 Legendre coefficients were used to represent the phase functions for each of 40 effective radius values ranging from 0.5 to $20 \mu \mathrm{m}$ in $0.5-\mu \mathrm{m}$ intervals. The surface was chosen to be perfectly absorbing.

Full 3D Monte Carlo computations were made for each of the 220 scenes for three solar zenith angles $\left(0^{\circ}\right.$, $30^{\circ}$, and $60^{\circ}$ ) and azimuthal angles of $0^{\circ}$ and $90^{\circ}$. The azimuthal angles are defined in Fig. 6 . The $0^{\circ}$ azimuth is defined as the solar input from the east, which is parallel to the stretching and tilting axes with the tops of the tilted clouds pointing toward this direction. The $90^{\circ}$ azimuth is broadside to the stretched and tilted clouds. A $270^{\circ}$ solar azimuth angle was not employed, as this gives results equivalent to the $90^{\circ}$ case for both the tilted and stretched fields. The $180^{\circ}$ azimuth indicates solar input from the west. As for $0^{\circ}$, this orientation is parallel to the axis of stretching and tilting and yields similar radiative results for the stretched fields. However, the tops of the tilted cloud fields point away from this direction, so calculations with a $180^{\circ}$ solar azimuth angle were performed for the vertically tilted cloud fields. Runs in which the photons were injected at random solar azimuth angles were also performed in order to investigate possible averaging effects due to variations in cloud-sun orientation over time or space.

In addition to the full $3 \mathrm{D}$ radiative transfer computations, IPA and TIPA calculations were carried out for the same scenes and solar zenith and azimuth angle combinations. These calculations were performed using the Monte Carlo algorithm by neglecting photon translation perpendicular to the vertical (IPA) or sunoriented (TIPA) atmospheric columns. The results of these approximate radiative transfer methods were compared to the output of the full 3D Monte Carlo to elucidate the ways in which anisotropic fields affect solar radiative propagation.

The variables provided by the Monte Carlo calculations were the domain-averaged downward direct and 


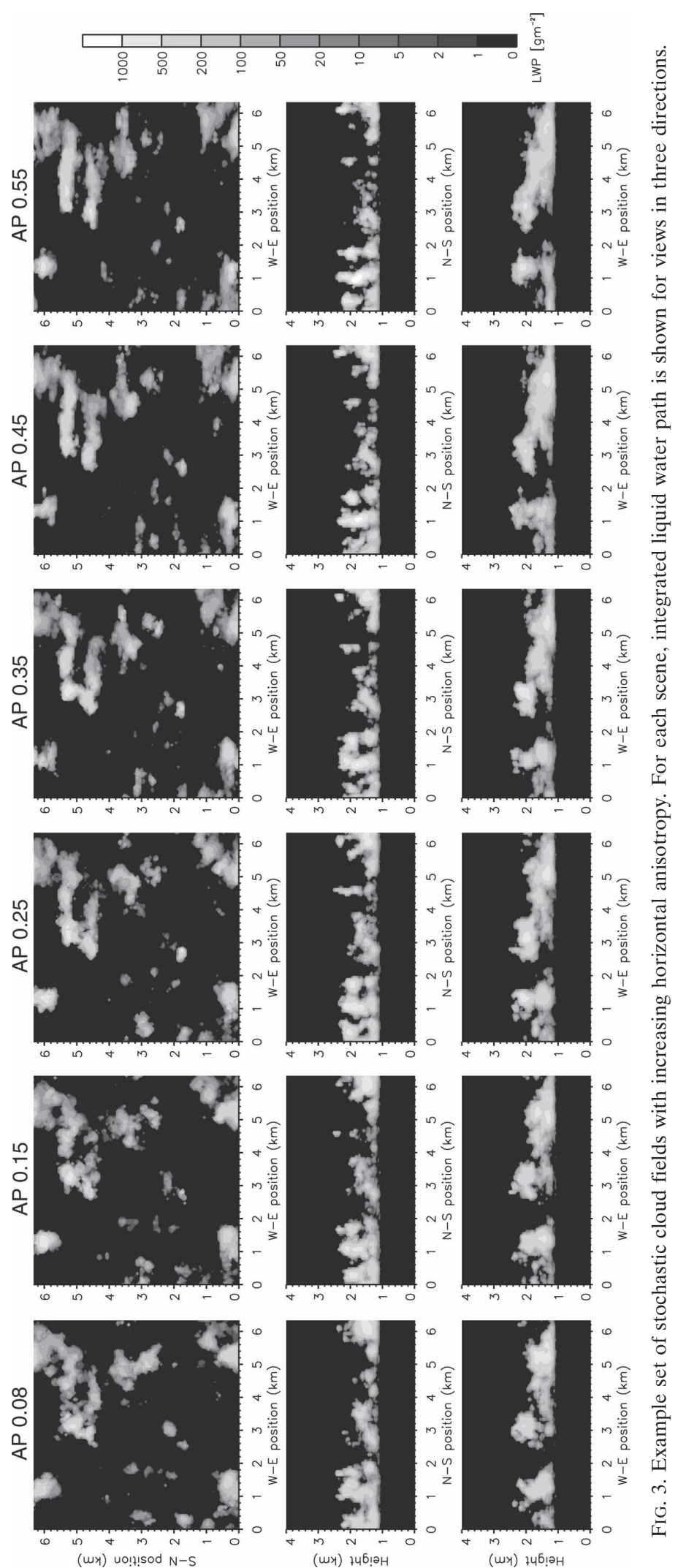




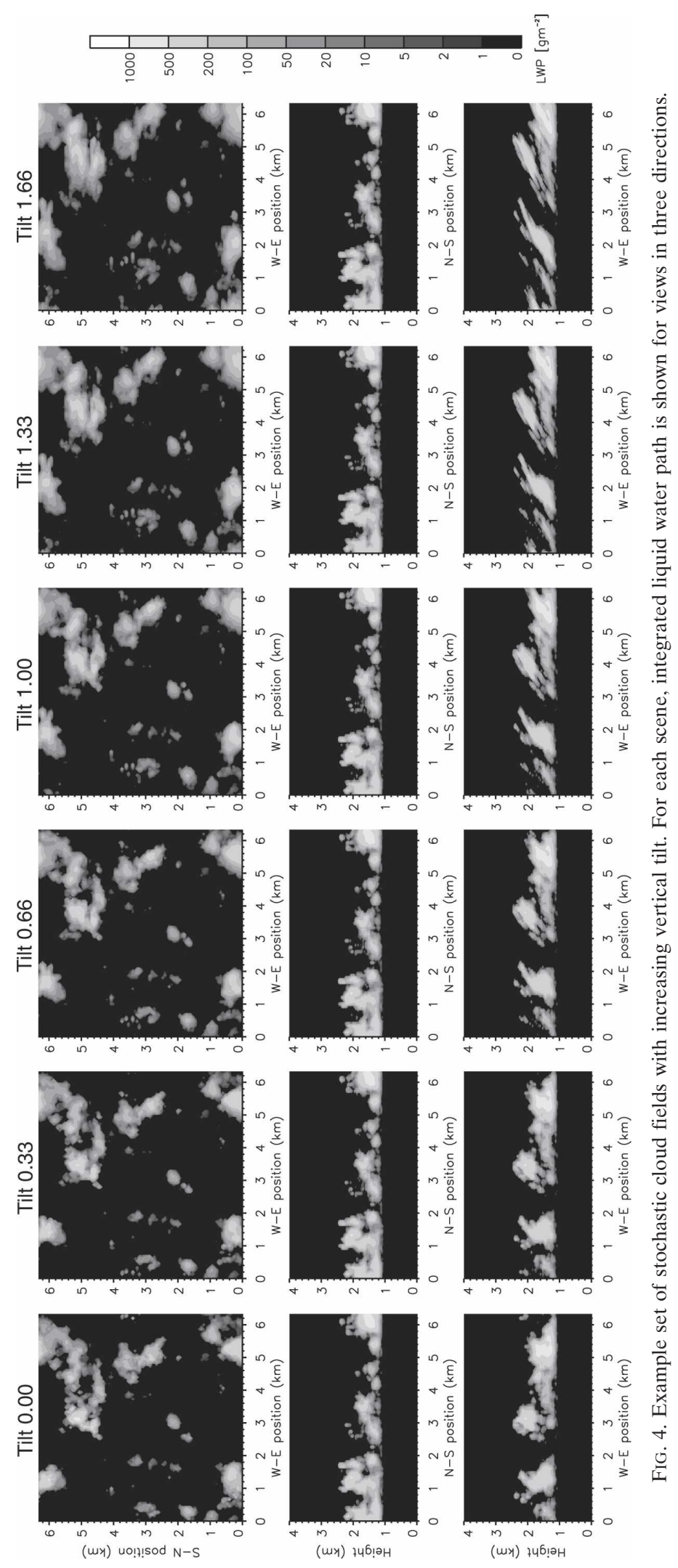


a
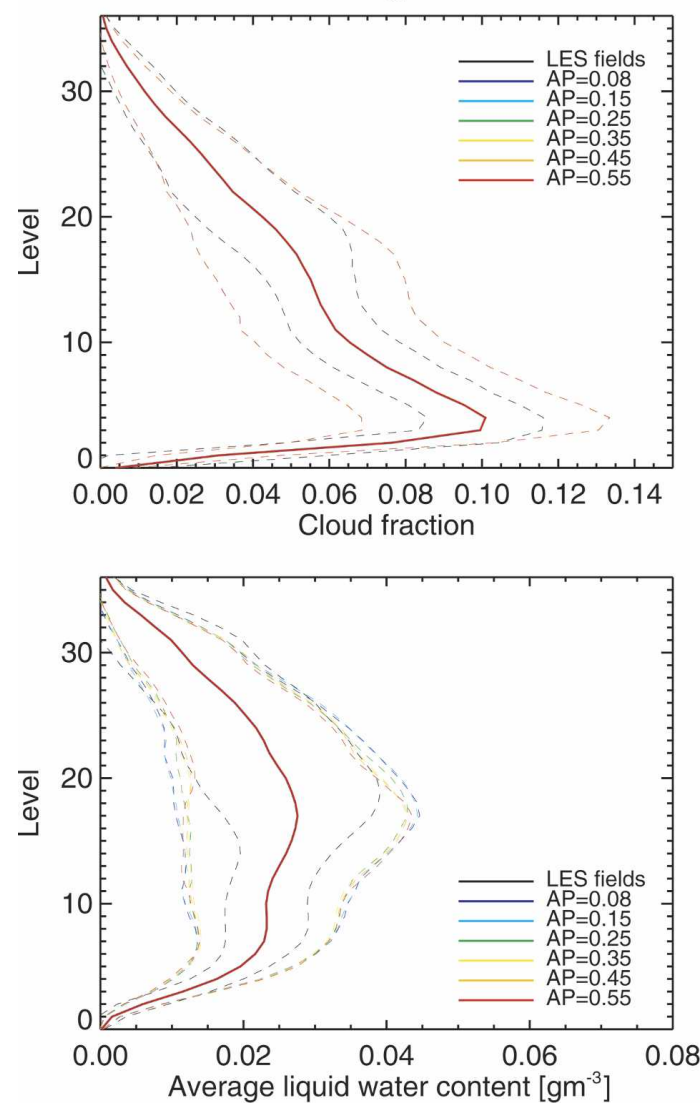

b
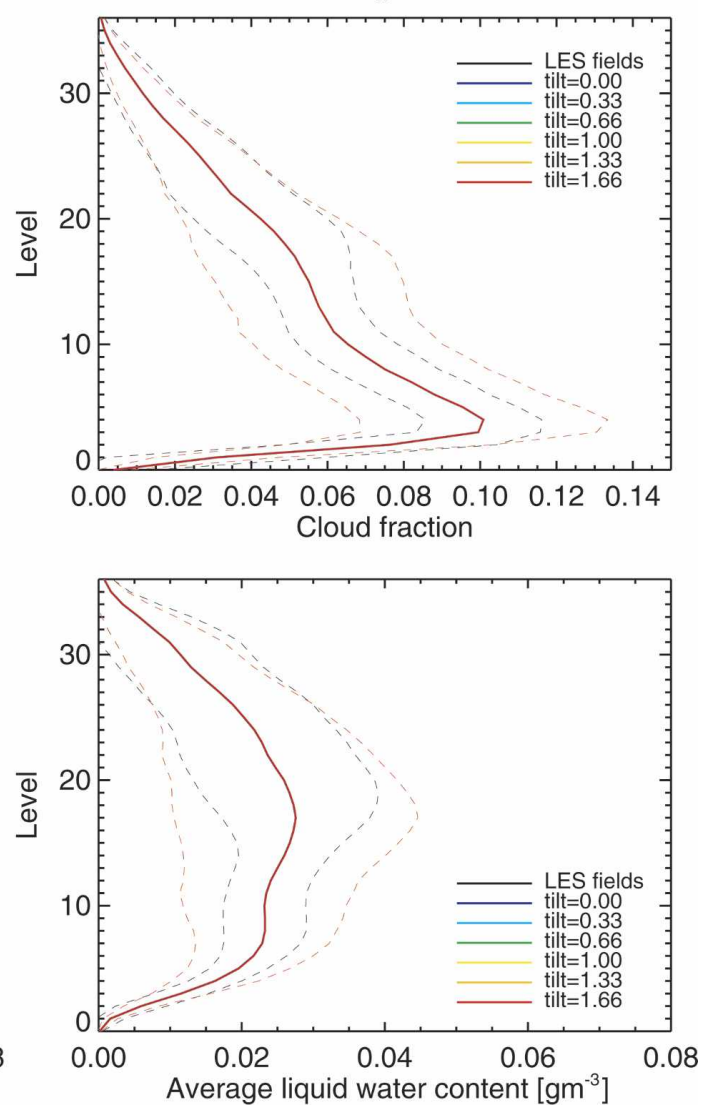

FIG. 5. Cloud fraction and average LWC as a function of height for input (LES) and stochastic fields for (a) horizontally stretched fields and (b) tilted fields. Solid lines represent ensemble means while dashed lines indicate the mean plus and minus $1 \mathrm{std}$ dev. Note that all of the mean value curves are coincident.

total surface fluxes, upward flux at the top of atmosphere, and total absorption. The diffuse ratio was computed as the difference between the total and direct surface fluxes normalized by the total downwelling surface flux. In addition, domain-averaged heating rate profiles were calculated. These heating rates were based on the average absorbed flux, temperature, and pressure at each model height. Six million photons were used for each Monte Carlo run. This number was selected based on accuracy and run time considerations. Tests run with a typical cloud field and a solar zenith angle of $30^{\circ}$ yielded the error estimates listed in Table 1.

\section{Results}

\section{a. Domain-averaged fluxes}

Domain-averaged transmission, reflection, absorption, and diffuse flux ratio as a function of cloud field horizontal anisotropy are presented in Fig. 7. Each point on the curves represents an average over the appropriate ensemble of 20 fields, while the error bars indicate the standard error of the mean for each ensemble. The top-left panel of Fig. 7 shows that cloud field anisotropy does affect the transmission of solar radiation for nonzero solar zenith angles. This effect depends on the orientation of the incident beam relative to the axis of cloud field stretching. For solar input parallel to the long axis of the clouds the transmission increases, while it decreases for solar incidence broadside to the stretched fields. These effects are consistent with a change in effective cloud fraction due to the stretching for nonzero solar zenith angles. Stretching along the east-west axis increases the cloud cross section presented to solar fluxes traveling perpendicular to the $x$ axis and thus reduces the proportion of the solar flux reaching the surface. The opposite is true for fluxes with angles of incidence parallel to the stretching axis. Although it is not obvious from the graphs, the values in Table 2 demonstrate that the impact of the anisotropy is small but nonzero for random solar azimuth angles. This is because the increase in transmission for incident angles parallel to the stretching axis is smaller 


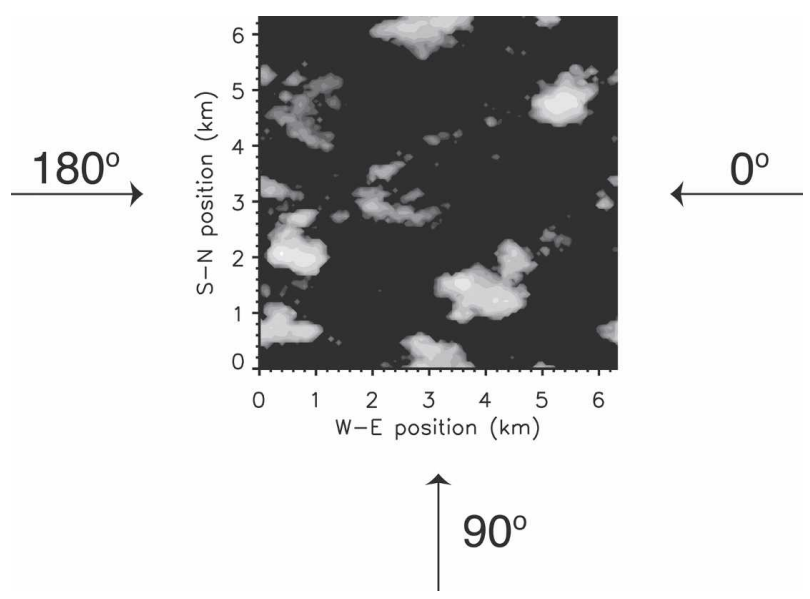

FIG. 6. Definition of solar azimuthal angles for Monte Carlo calculations. Azimuths of $0^{\circ}$ and $180^{\circ}$ are parallel to the axis of distortion in all scenes, while $90^{\circ}$ is broadside to this axis. Clouds tilt toward $0^{\circ}$ and away from $180^{\circ}$.

than the decrease in transmission that occurs for broadside incidence. The fact that increasing horizontal anisotropy has relatively little effect for overhead sun confirms that cloud properties are well conserved as the anisotropy is increased.

Results for reflection or albedo, shown in the upperright panel of Fig. 7, are essentially the inverse of the transmission results. The albedo increases with increasing anisotropy when the solar flux is incident on the side of the clouds and decreases with increasing anisotropy when the incoming flux is parallel to the stretching axis. Changes in the total absorption are small (note the change in vertical scale and the values listed in Table 3), but vary in direction according to the effective cloud fraction [i.e., absorption increases (decreases) with anisotropy for side (parallel) solar incidence]. The largest relative changes with anisotropy in Fig. 7 occur in the diffuse ratio (bottom-right panel). As we would expect, the diffuse ratio increases with increasing effective cloud fraction and vice versa.

Domain-averaged transmission, reflection, absorption, and diffuse ratio as a function of cloud tilt are presented in Fig. 8 and the transmitted flux values are listed in Table 4. In general, the effect of tilt is larger than that of stretching for most solar geometries. Even for a solar zenith angle of $0^{\circ}$, tilt significantly increases the albedo and decreases transmission. This is because shifting cloud layers horizontally causes an increase in cloud fraction for overhead sun, while cloud fraction is conserved in the stretching process. Apparent cloud fraction also increases with tilt for the $90^{\circ}$ azimuth (broadside to the tilting axis). Thus, the transmission decreases with increasing tilt from this direction as well.

It is noteworthy that the transmission values calcu-
TABLE 1. Monte Carlo noise estimates for individual 3D runs.

\begin{tabular}{ccccc}
\hline \hline \multicolumn{2}{c}{ Domain-averaged values } & & \multicolumn{2}{c}{ Absorption profiles } \\
\cline { 1 - 2 } \cline { 5 - 5 } Quantity & Error & & Quantity & Error \\
\hline $\begin{array}{c}\text { Downward surface } \\
\text { flux }\end{array}$ & $0.03 \%$ & & $\begin{array}{c}\text { Average value in } \\
\text { cloud }\end{array}$ & $1.0 \%$ \\
$\begin{array}{c}\text { Upward flux, top of } \\
\text { atmosphere } \\
\text { Total absorption }\end{array}$ & $0.13 \%$ & & $\begin{array}{c}\text { Cloudy region } \\
\text { maximum }\end{array}$ & $1.4 \%$ \\
\hline
\end{tabular}

lated for solar incidence parallel to the stretching axis (azimuths of $0^{\circ}$ and $180^{\circ}$ ) are very different in the presence of tilted fields. For a $180^{\circ}$ azimuthal angle, the clouds tilt away from the incoming radiation, thus presenting a greater cross section to the incident flux and decreasing solar transmission. In contrast, for the $0^{\circ}$ azimuth, the clouds tilt toward the sun, which reduces their effective cross section. The maximum transmission for tilted cloud fields occurs at the $0^{\circ}$ azimuth when the tilt of the clouds matches the solar zenith angle, so that the effective cloud fraction is minimized. For the $30^{\circ}$ solar zenith angle, this occurs at a tilt of about 0.66 . The transmission peaks at this tilt increment, then decreases as the tilt continues to grow. No peak is observed in the $60^{\circ}$ solar zenith angle transmission curve because the imposed tilts do not exceed $60^{\circ}$.

In contrast to horizontal stretching, the impact of tilt on absolute fluxes generally diminishes for larger solar zenith angles (see Table 4). This is because the $60^{\circ}$ solar zenith angle is nearly a side view; lateral displacements of cloud structures do not change the total crosssectional area greatly from this perspective. Only for the tilt-aligned $0^{\circ}$ azimuth is the largest effect on the downward flux observed for the $60^{\circ}$ solar zenith angle.

As for the horizontally anisotropic fields, the changes in the albedo with tilt are nearly opposite the differences found for transmission. Again, the variations in total absorption, listed in Table 5, are small but nonzero. Except for the $0^{\circ}$ azimuth, the diffuse ratio always increases with tilt, reaching values over 0.4 for the most tilted scenes at low sun angles.

Again as with the stretched cases, use of random azimuthal angles does not "average out" the effects of tilt. The reason for this is evident from the differences in transmitted flux relative to the untilted scenes listed in Table 4. Increasing tilt decreases the transmission (increases the albedo) for all azimuthal angles except $0^{\circ}$ and the effect at $0^{\circ}$ is not enough to outweigh the contributions from the other solar azimuthal angles.

From Tables 2 and 4 it is apparent that not taking horizontal anisotropy into account can cause errors in the downward flux at the surface close to $10 \mathrm{~W} \mathrm{~m}^{-2}$, while neglecting vertical tilt can lead to errors as high as 

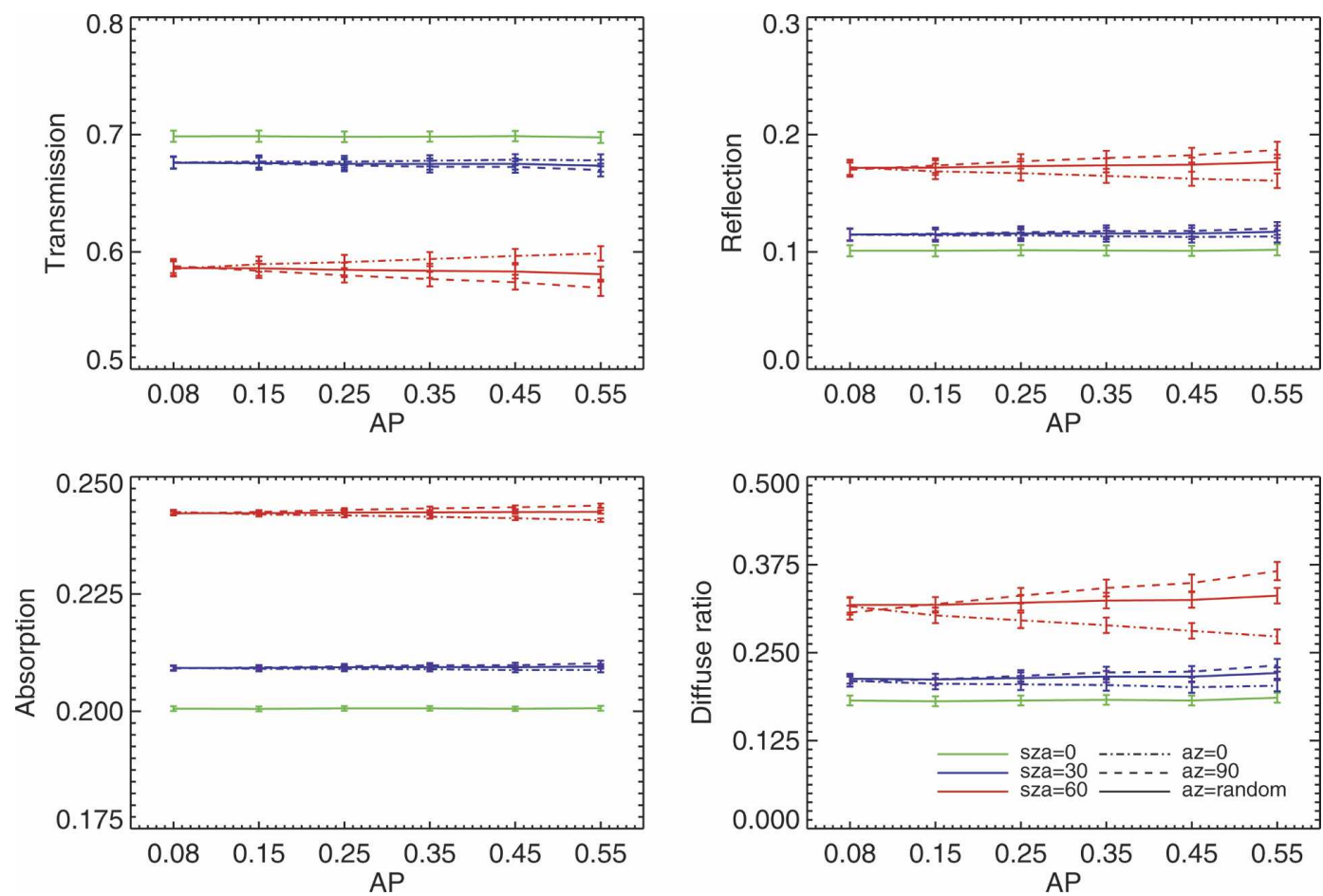

FIG. 7. Domain-averaged (top left) transmission, (top right) reflection, (bottom left) absorption, and (bottom right) diffuse flux ratio for cloud scenes with increasing horizontal anisotropy. Error bars indicate the standard error of the mean over the 20 -scene ensembles.

$40 \mathrm{~W} \mathrm{~m}^{-2}$ in extreme cases. Errors of this magnitude can be important to energy budget calculations if they persist for an extended length of time. The errors in the absorbed flux are much smaller in magnitude, but still significant as a fraction of the total fluxes.

\section{b. Atmospheric heating rate profiles}

Although the changes observed in domain-averaged absorption due to cumulus tilt or anisotropy were small, their spatial distribution could have important implications for the local energy budget or the dynamics of the cloud fields. For this reason, we analyze the heating rate changes caused by cloud field anisotropy as a function of height in the domain.

The change in heating rate profiles for cloud scenes as vertical tilt increases is shown in Fig. 9. A separate plot is shown for each solar zenith angle-azimuth angle combination. The heating rate profile for the ensemble of untilted cases is illustrated by the black line. Differences between this profile and those for the progressively more tilted cases are shown in various shades of gray. The general shape of the base heating rate curve is determined by the distribution of liquid water with height. Since most of the absorption occurs within

TABLE 2. Differences between downward surface fluxes computed for stretched and isotropic cloud cases $\left(\mathrm{W} \mathrm{m}^{-2}\right)$.

\begin{tabular}{|c|c|c|c|c|c|c|c|}
\hline \multirow{3}{*}{$\begin{array}{l}\text { Solar zenith } \\
\text { angle }\end{array}$} & \multirow[b]{3}{*}{ Azimuth angle } & \multicolumn{6}{|c|}{ Anisotropy } \\
\hline & & \multirow{2}{*}{$\frac{0.08}{\text { Flux }}$} & 0.15 & 0.25 & 0.35 & 0.45 & 0.55 \\
\hline & & & \multicolumn{5}{|c|}{ Downward flux difference } \\
\hline $0^{\circ}$ & Random & 955.7 & 0.1 & -0.7 & -0.4 & 0.2 & -1.2 \\
\hline \multirow[t]{3}{*}{$30^{\circ}$} & $0^{\circ}$ & 801.0 & 1.1 & 0.9 & 2.0 & 3.0 & 2.4 \\
\hline & $90^{\circ}$ & 801.3 & -1.3 & -3.0 & -4.4 & -4.7 & -7.8 \\
\hline & Random & 800.9 & -0.2 & -1.1 & -1.2 & -1.1 & -3.0 \\
\hline \multirow[t]{3}{*}{$60^{\circ}$} & $0^{\circ}$ & 400.5 & 2.7 & 3.9 & 5.7 & 7.6 & 9.0 \\
\hline & $90^{\circ}$ & 402.2 & -2.7 & -5.4 & -7.6 & -9.4 & -12.7 \\
\hline & Random & 401.0 & -0.1 & -1.1 & -1.6 & -2.0 & -3.5 \\
\hline
\end{tabular}


TABLE 3. Differences between domain-averaged absorbed fluxes for stretched cloud and isotropic cases $\left(\mathrm{W} \mathrm{m}^{-2}\right)$.

\begin{tabular}{|c|c|c|c|c|c|c|c|}
\hline \multirow{3}{*}{$\begin{array}{l}\text { Solar zenith } \\
\text { angle }\end{array}$} & \multirow[b]{3}{*}{ Azimuth angle } & \multicolumn{6}{|c|}{ Anisotropy } \\
\hline & & 0.08 & 0.15 & 0.25 & 0.35 & 0.45 & 0.55 \\
\hline & & Absorbed flux & \multicolumn{5}{|c|}{ Absorbed flux difference } \\
\hline $0^{\circ}$ & Random & 274.4 & -0.1 & 0.1 & 0.1 & -0.0 & 0.1 \\
\hline \multirow[t]{3}{*}{$30^{\circ}$} & $0^{\circ}$ & 248.0 & -0.3 & -0.3 & -0.4 & -0.6 & -0.6 \\
\hline & $90^{\circ}$ & 247.8 & 0.2 & 0.5 & 0.8 & 0.8 & 1.3 \\
\hline & Random & 247.9 & -0.0 & 0.2 & 0.2 & 0.2 & 0.4 \\
\hline \multirow[t]{3}{*}{$60^{\circ}$} & $0^{\circ}$ & 165.9 & -0.4 & -0.5 & -0.7 & -0.9 & -1.2 \\
\hline & $90^{\circ}$ & 165.6 & 0.3 & 0.6 & 0.8 & 0.9 & 1.2 \\
\hline & Random & 165.7 & 0.0 & 0.1 & 0.1 & 0.2 & 0.2 \\
\hline
\end{tabular}

the first few optical depths of absorbers encountered by the photons, the heating rate maximum occurs near the cloud top. The heating rate is then fairly constant throughout the depth of the clouds before tapering off below the cloud base.

From the perspective of atmospheric heating rates, the effect of tilt is to alter the apparent cloud-top area. For example, for overhead sun, skewing enlarges the upper surface area of the cloud field. This causes heating rates to rise at all but the top levels because new upward surfaces are exposed at lower elevations as the sides of the clouds peak out from below. This same effect occurs for all solar azimuthal and zenith angles except for the azimuth of $0^{\circ}$. For this geometry, increas- ing tilt decreases the apparent cloud fraction, reducing the cloud-top area and heating rates correspondingly. The occurrence of a position of minimum cloud fraction is apparent in the curves for a solar zenith angle $30^{\circ}$ and azimuth of $0^{\circ}$. Starting from no tilt, the heating rates first decrease and then increase after the minimum in apparent cloud-top surface area is reached at a tilt of 0.66 . Although a similar decrease in heating rate with increased tilt is observed for the $60^{\circ}$ solar zenith angle, a minimum is not reached because of an insufficient tilt in the cloud fields. The maximum local heating rate change caused by tilt in this study is $+0.65 \mathrm{~K} \mathrm{day}^{-1}$, corresponding to an increase of $17 \%$. This occurs at the top of the shoulder region of the profile (at $2.22 \mathrm{~km}$ ) for
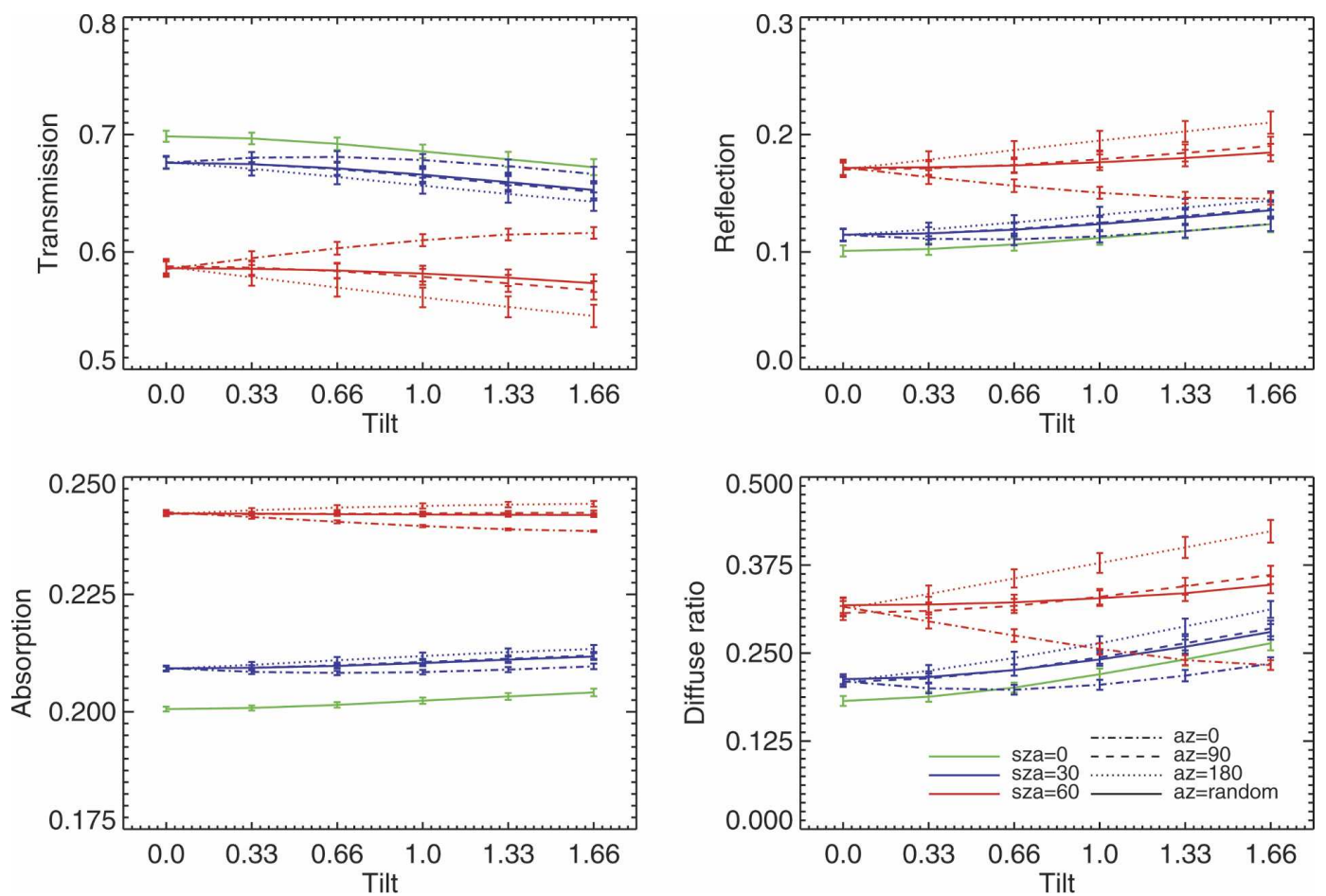

FIG. 8. Same as Fig. 7 except for cloud scenes with increasing vertical tilt. 
TABLE 4. Differences between downward surface fluxes computed for tilted and untilted cloud cases $\left(\mathrm{W} \mathrm{m}^{-2}\right)$.

\begin{tabular}{|c|c|c|c|c|c|c|c|}
\hline \multirow{3}{*}{$\begin{array}{l}\text { Solar zenith } \\
\text { angle }\end{array}$} & \multirow[b]{3}{*}{ Azimuth angle } & \multicolumn{6}{|c|}{ Tilt } \\
\hline & & \multirow{2}{*}{$\frac{0.0}{\text { Flux }}$} & 0.33 & 0.66 & 1.0 & 1.33 & 1.66 \\
\hline & & & \multicolumn{5}{|c|}{ Downward flux difference } \\
\hline $0^{\circ}$ & Random & 955.8 & -2.5 & -8.7 & -17.6 & -26.8 & -36.0 \\
\hline \multirow[t]{4}{*}{$30^{\circ}$} & $0^{\circ}$ & 801.0 & 5.1 & 5.9 & 2.7 & -3.3 & -11.3 \\
\hline & $90^{\circ}$ & 801.4 & -1.8 & -6.9 & -14.0 & -21.7 & -29.8 \\
\hline & $180^{\circ}$ & 801.7 & -6.8 & -14.9 & -23.8 & -32.2 & -39.9 \\
\hline & Random & 800.9 & -1.3 & -5.6 & -12.0 & -19.6 & -27.5 \\
\hline \multirow[t]{4}{*}{$60^{\circ}$} & $0^{\circ}$ & 400.5 & 6.4 & 12.2 & 16.9 & 20.2 & 21.1 \\
\hline & $90^{\circ}$ & 402.1 & -0.8 & -2.9 & -6.2 & -10.0 & -14.1 \\
\hline & $180^{\circ}$ & 401.9 & -6.2 & -12.2 & -17.9 & -23.5 & -28.8 \\
\hline & Random & 401.0 & -0.3 & -1.3 & -3.1 & -5.6 & -8.7 \\
\hline
\end{tabular}

the $30^{\circ}$ solar zenith angle $-180^{\circ}$ solar azimuth angle combination.

Whether the effects of tilt on atmospheric heating rate profiles can be cancelled out by variations in azimuthal angle depends on the solar zenith angle. For solar zenith angles of $0^{\circ}$ and $30^{\circ}$, heating rates increase with tilt as the apparent cloud fraction increases even for random azimuthal angles. But at the $60^{\circ}$ solar zenith angle, there is a great deal of side incidence at all azimuthal angles, so the changes in heating rate with cloud tilt are of lesser magnitude. The strong decrease in heating rates with tilt for the $0^{\circ}$ azimuth more than offsets the increase for $180^{\circ}$. With the addition of small heating rate increases for azimuths of perpendicular solar incidence, there is nearly no net change in the atmospheric heating rate profile at a $60^{\circ}$ zenith angle when random azimuthal angles are employed.

The effect of horizontal anisotropy on atmospheric heating rate profiles is illustrated in Fig. 10. Again, there is a separate graph for each zenith-azimuth angle combination and the shade of the lines indicates the level of anisotropy in the ensemble represented. Note that there are fewer graphs because the $180^{\circ}$ azimuth is not considered.

The behavior of the heating rate profiles with increasing horizontal anisotropy is as follows. For overhead sun, almost no change is observed because the cloud fraction and vertical optical depth distributions are well conserved as the clouds are stretched. For more oblique angles of incidence, heating rates decline for solar azimuthal angles parallel to the stretching axis $\left(0^{\circ}\right)$. For these relative solar positions, the apparent optical depths rise but the cloud cross sections decline, giving less effective cloud-top area. The opposite occurs for solar incidence perpendicular to the stretching axis where the apparent optical depths decrease but the cloud-top surface area increases, yielding higher heating rates. For this reason, the changes in the heating rate profiles due to horizontal anisotropy tend to cancel for random azimuthal angles. Horizontal anisotropy has a significantly smaller impact on heating rate profiles than vertical tilt does for the scenes used in this study. The maximum heating rate profile change caused by horizontal anisotropy is only $+0.25 \mathrm{~K} \mathrm{day}^{-1}$. However, this occurs for a solar zenith angle of $60^{\circ}$ and

TABLE 5. Differences between domain-averaged absorbed fluxes for tilted and untilted cloud cases $\left(\mathrm{W} \mathrm{m} \mathrm{m}^{-2}\right)$.

\begin{tabular}{|c|c|c|c|c|c|c|c|}
\hline \multirow{3}{*}{$\begin{array}{l}\text { Solar zenith } \\
\text { angle }\end{array}$} & \multirow[b]{3}{*}{ Azimuth angle } & \multicolumn{6}{|c|}{ Tilt } \\
\hline & & 0.0 & 0.33 & 0.66 & 1.0 & 1.33 & 1.66 \\
\hline & & Absorbed flux & \multicolumn{5}{|c|}{ Absorbed flux difference } \\
\hline $0^{\circ}$ & Random & 274.4 & 0.3 & 1.2 & 2.5 & 3.7 & 4.9 \\
\hline \multirow[t]{4}{*}{$30^{\circ}$} & $0^{\circ}$ & 248.0 & -1.0 & -1.2 & -1.0 & -0.4 & 0.4 \\
\hline & $90^{\circ}$ & 247.8 & 0.3 & 0.9 & 1.7 & 2.6 & 3.4 \\
\hline & $180^{\circ}$ & 247.8 & 1.0 & 2.2 & 3.2 & 4.2 & 5.0 \\
\hline & Random & 247.9 & 0.2 & 0.6 & 1.3 & 2.2 & 3.0 \\
\hline \multirow[t]{4}{*}{60} & $0^{\circ}$ & 165.9 & -0.7 & -1.4 & -2.0 & -2.5 & -2.8 \\
\hline & $90^{\circ}$ & 165.6 & -0.0 & 0.0 & 0.1 & 0.2 & 0.2 \\
\hline & $180^{\circ}$ & 165.6 & 0.6 & 1.0 & 1.2 & 1.4 & 1.5 \\
\hline & Random & 165.7 & -0.0 & -0.1 & -0.2 & -0.2 & -0.2 \\
\hline
\end{tabular}



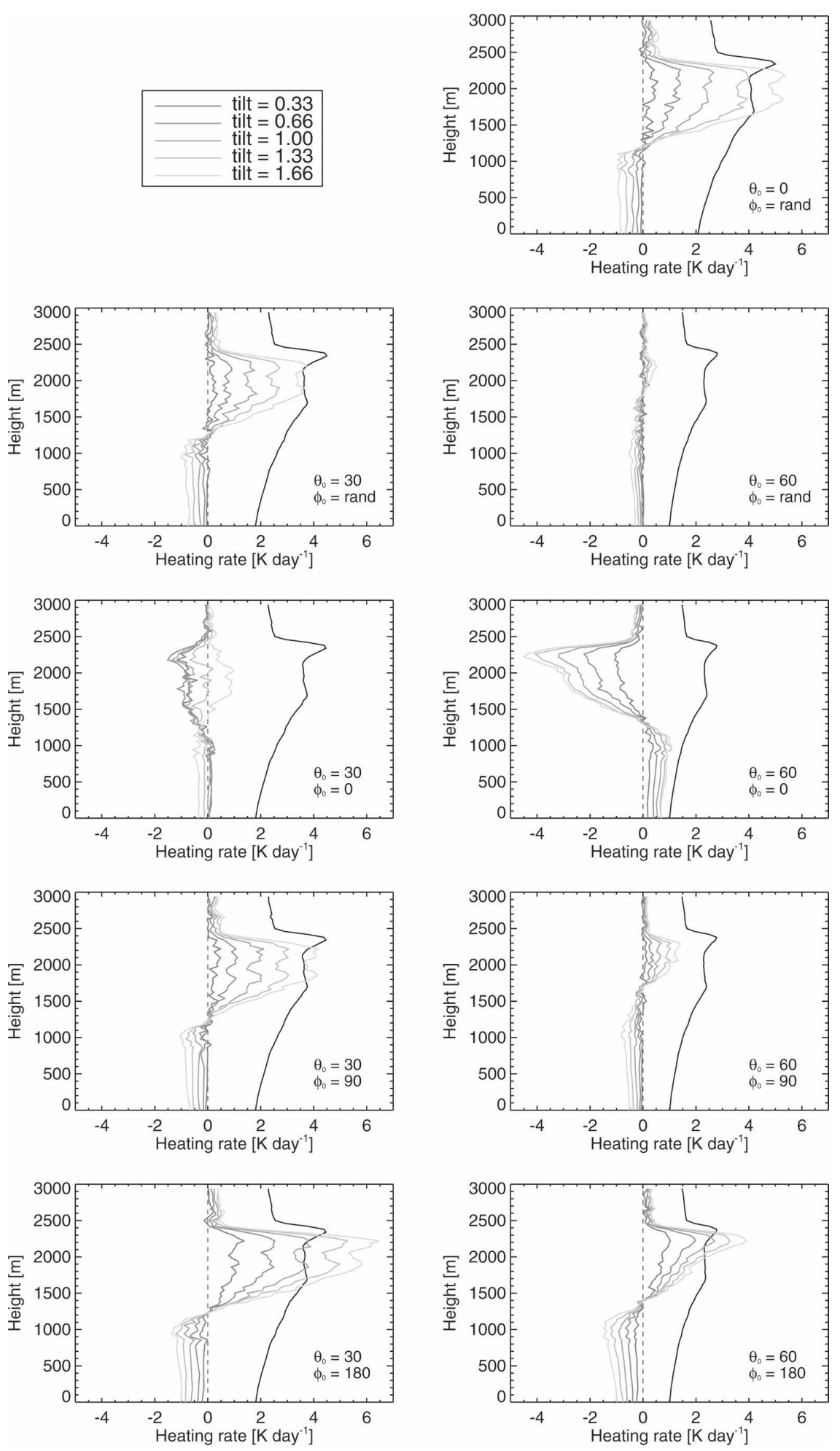

FIG. 9. Differences between heating rate profiles for tilted and untilted cloud cases. In each panel, the black curve represents the mean heating rate profile for the untilted cases and corresponds to the values listed on the $x$ axis. The remaining curves indicate 10 times the difference between the mean heating rates for the tilted and untilted cases; $\theta_{0}$ indicates the solar zenith angle and $\phi_{0}$ the solar azimuth angle. 

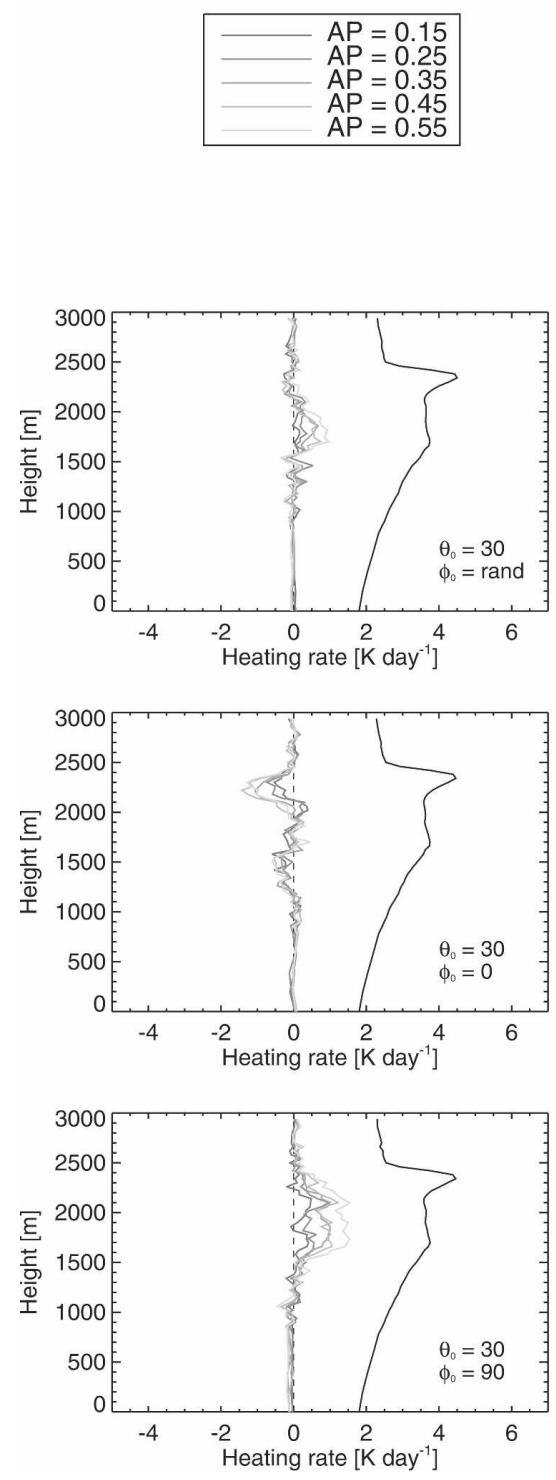
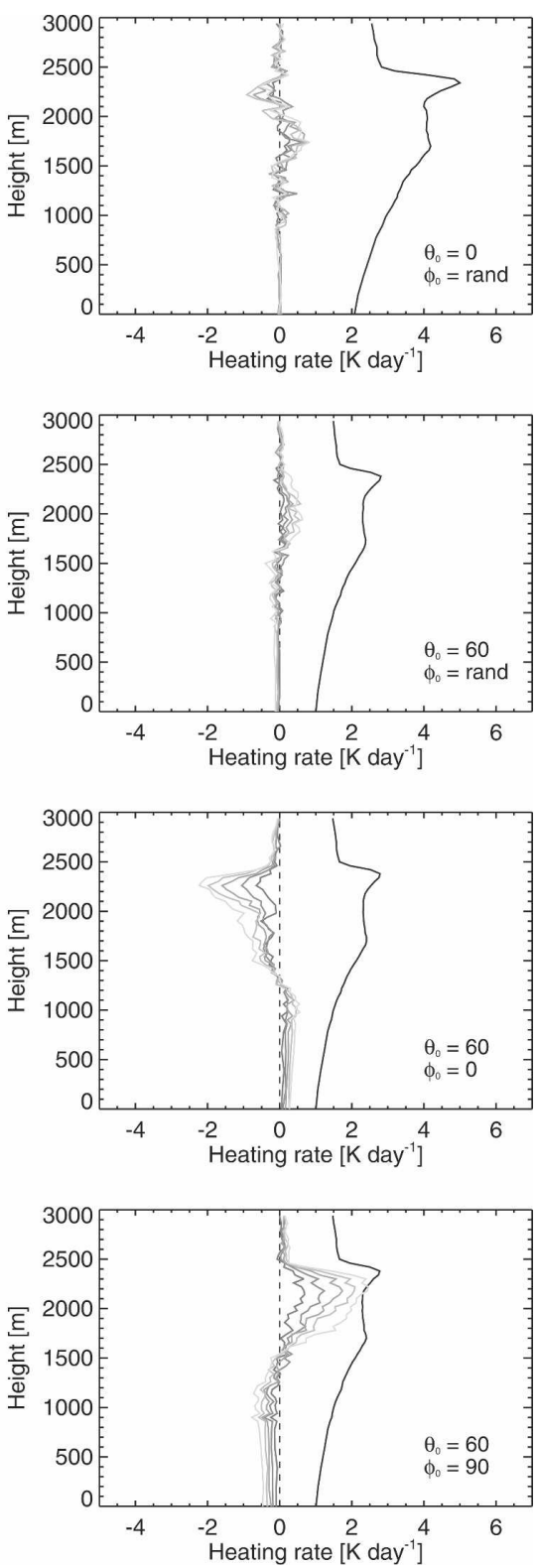

FIG. 10. Same as Fig. 9 except for stretched and isotropic cloud cases.

azimuth of $90^{\circ}$, so that the relative change is more than $10 \%$. This maximum also occurs at $2.22 \mathrm{~km}$.

\section{Discussion}

\section{a. Mechanisms by which anisotropy affects solar radiative transfer}

Comparing results for the IPA, TIPA, and full 3D radiative transfer calculations illuminates the processes by which anisotropy affects solar radiative transport since each successive approximation further restricts the accuracy of photon tracing through the cloud scenes. Differences between the IPA and 3D results reflect both parts of the $3 \mathrm{D}$ effect-constraining the photons to stay within a given vertical column eliminates scattering into neighboring columns and neglects the changes in apparent cloud geometry for nonvertical solar illumination. TIPA, on the other hand, accounts for the apparent changes in cloud geometry as a function of solar incidence. Differences between TIPA and $3 \mathrm{D}$ results thus stem entirely from the neglect of scattering perpendicular to these paths.

Differences between the transmitted fluxes for IPA and 3D computations for the stretched and tilted scenes 

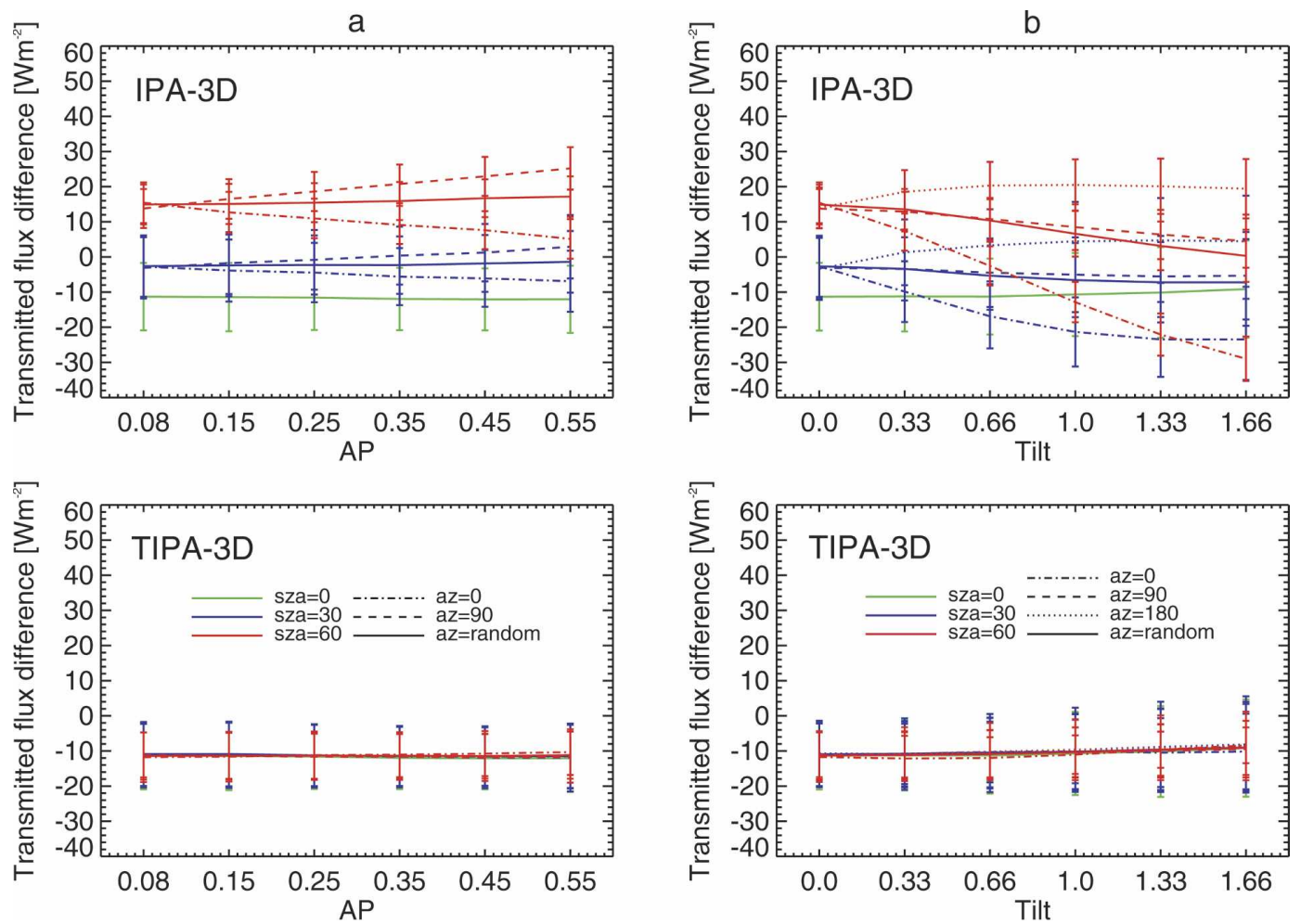

FIG. 11. Differences between domain-averaged transmitted fluxes computed using (top) IPA or (bottom) TIPA and full 3D computations for cloud scenes with increasing levels of (a) horizontal anisotropy and (b) vertical tilt. Error bars indicate the standard error of the mean over the 20 -scene ensembles.

are shown in the top panels of Fig. 11. Neglect of both types of $3 \mathrm{D}$ effects leads to errors as large as $23 \mathrm{~W} \mathrm{~m}^{-2}$ for the stretched cases and $29 \mathrm{~W} \mathrm{~m}^{-2}$ for the tilted cases. Since the $3 \mathrm{D}$ results are highly dependent on solar zenith and azimuth angles while the IPA is nearly insensitive to solar position, the IPA errors depend strongly on the relative sun angles. For the stretched scenes, the IPA errors for $0^{\circ}$ and $90^{\circ}$ tend to be offsetting, but even for random azimuthal angles, the IPA results still differ from the full $3 \mathrm{D}$ transmitted fluxes. The same is true for the $0^{\circ}$ and $180^{\circ}$ azimuth results for the tilted fluxes. For both the tilted and stretched scenes, only the fluxes for the $30^{\circ}$ solar zenith angle and random (also $90^{\circ}$ for the tilted scenes) azimuth angles stay close to $0 \mathrm{~W} \mathrm{~m}^{-2}$ as the degree of anisotropy increases.

The differences between the TIPA and full 3D transmitted fluxes, illustrated in the bottom panels of Fig. 11, indicate that changes in cloud geometry are the chief driver of the variations in domain-averaged solar radiative quantities that we have observed with increasing anisotropy. Since both the TIPA and 3D computations use the proper cloud geometry for each direction of solar incidence, the differences between the results of these methods are primarily due to the omission of scat- tering out of the direction of incidence by the TIPA algorithm. Figure 11 shows that the magnitude of the perpendicular transport is nearly constant regardless of tilt, horizontal anisotropy, and even relative solar position, having mean values between 9 and $12 \mathrm{~W} \mathrm{~m}^{-2}$ in all cases. This suggests that the perpendicular transport is largely a function of the basic properties (such as LWC, cloud amount, and size) of clouds in a scene and only minimally impacted by minor changes in cloud geometry.

Changes in apparent cloud geometry due to relative solar position can be further divided into changes in effective cloud fraction and mean cloud optical depth. Cloud fraction is a measure of the extent of a cloud field while optical depth is related to the intensity or duration of the interaction between the solar photons and cloud droplets. Effective cloud fraction and effective optical depth were computed for each stochastic cloud field by Monte Carlo sampling of photons injected at the desired solar zenith and azimuth angles. For the correct (3D) relative position of the sun, the effective cloud fraction curves (not shown) precisely mirror the albedo curves in Figs. 7 and 8, while the mean cloud optical depths change in the opposite direction as anisotropy increases. Note that the observed 

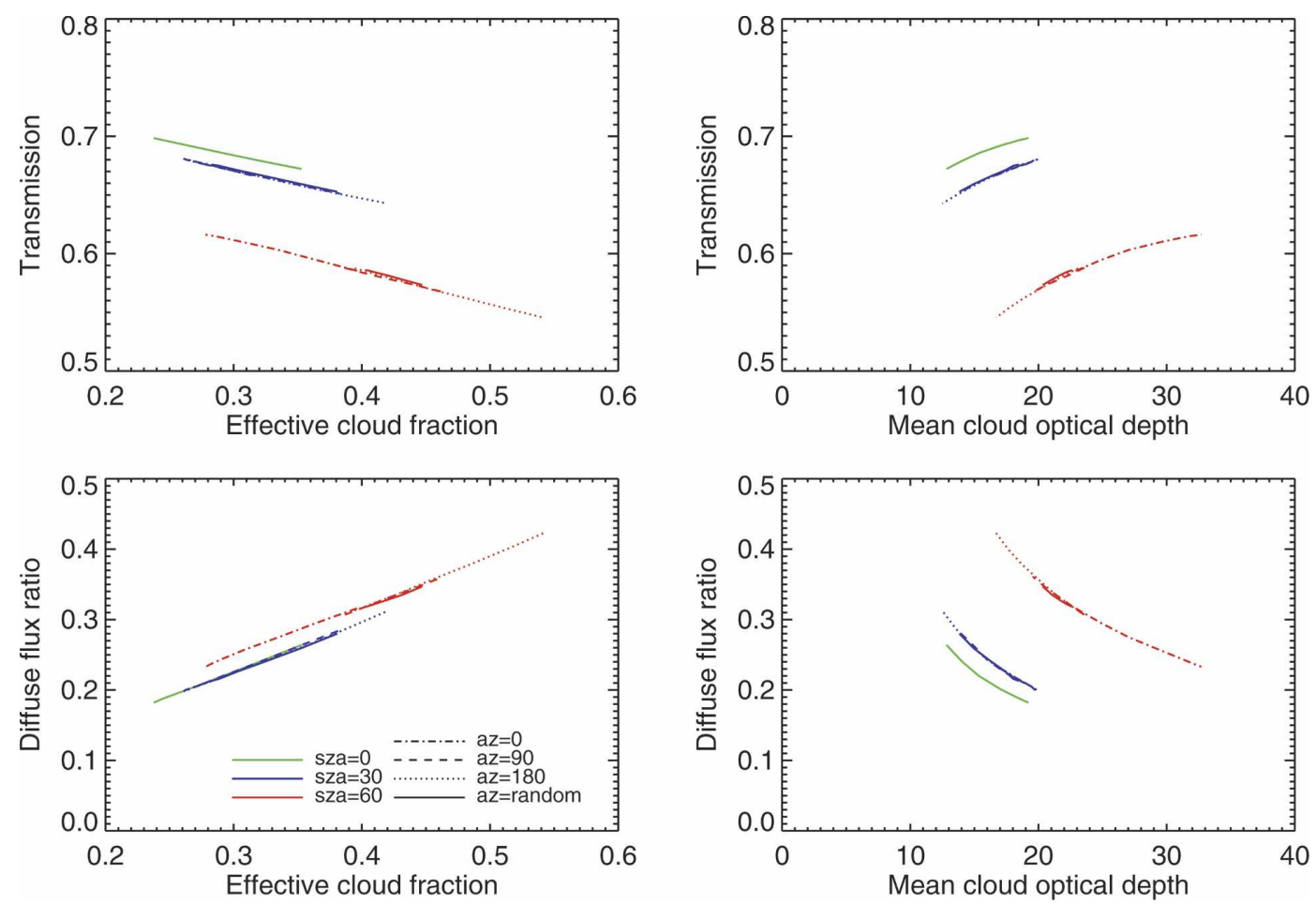

FIG. 12. Domain-averaged (top) transmission and (bottom) diffuse flux ratio for tilted cloud fields as a function of (left) effective cloud fraction and (right) mean cloud optical depth. (Full 3D computations.)

inverse relationship between apparent cloud fraction and optical depth is a special characteristic of the fields used in this study that arises because the total LWC of the cloud fields is held fixed as anisotropy increases.

Earlier, we argued that the changes in solar flux terms computed for cloud scenes with increasing levels of anisotropy are consistent with changes in effective cloud fraction. This hypothesis is supported by the plots of the transmission and diffuse flux ratio as a function of the effective cloud fraction and optical depth shown in Fig. 12. Other things being equal, we would expect transmission to decrease as cloud fraction or optical depth increase. While this is the case for the effective cloud fraction, transmission is observed to increase as optical depth increases. This implies that, at least for the cloud scenes studied here, effective cloud fraction has a greater impact on transmitted fluxes than does cloud optical depth. For the diffuse flux ratio, the expected trends are opposite those for transmission, that is, diffuse flux ratio should increase as either effective cloud fraction or optical depth increase. Again, we find that the expectation holds true for cloud fraction but not for optical depth. By physical reasoning, then, the observed changes in the solar fluxes must be due to variations in cloud cover rather than changes in mean cloud optical depth caused by increasing cloud anisotropy. (Note that only results for the tilted scenes are shown in this figure. The trends for the stretched scenes are the same, but the cloud fractions and optical depths fall within a more limited range.)

Although the effective optical depth seems to play little part in the changes in the domain-averaged radiative transfer associated with increasing anisotropy, it is relevant to the absolute magnitude of the domainaveraged fluxes as a function of the solar zenith angle. The large separation between the transmission curves as a function of the effective cloud fraction in Fig. 12 are explained by the overall increase in optical depth as the solar zenith angle increases. In contrast, the computed diffuse flux ratios have nearly a 1:1 correspondence with the corresponding effective cloud fraction values, suggesting that the diffuse flux ratios for the scenes considered here are almost entirely a function of the effective cloud fraction alone.

We conclude that tilt and horizontal anisotropy affect solar radiative transport primarily by changing the effective cloud fraction of a scene. Changes in the mean optical path are of lesser importance. Transport perpendicular to the direction of incidence is of a significant magnitude for the cases examined here, but is largely unchanged by simple rearrangements of the 
LWC distributions that leave the basic cloud properties unchanged.

\section{b. Potential approximate methods of radiative transfer for anisotropic cumulus clouds}

Given the results of the various radiative transfer computations described above, we can consider the effectiveness of various treatments of cumulus anisotropy in modeling and measurement situations. There are two factors that control the accuracy of such treatments: the amount of cloud structure information that is available and the degree of approximation in the radiative transfer algorithm employed. If the complete 3D structure of the cloud field is known, IPA, TIPA, and full 3D radiative transfer methods are all applicable. Of course the 3D computations will give accurate flux and heating rate values. However, if a faster method is desired, applying TIPA will be reasonably accurate. As discussed previously, the error in transmitted fluxes computed using TIPA is always negative and on the order of $10 \mathrm{~W}$ $\mathrm{m}^{-2}$ for the type of scenes used here. For other cloud fields, the size of the bias could be estimated from known properties of the cloud fields. This would require previous computations of sample scenes with various values of mean cloud size and optical depth, cloud thickness, and average spacing between clouds. There is no advantage to using IPA because it gives widely varying and frequently large errors $(-30$ to 25 $\mathrm{W} \mathrm{m}{ }^{-2}$ for the scenes used here) while being no more computationally efficient than TIPA.

In most modeling and even measurement situations, the full 3D cloud field structure is not known at high resolution. The basic information needed as input to TIPA for anisotropic cumulus fields is the optical depth distribution as a function of the solar zenith and azimuth angles [i.e., $\left.P\left(\tau \mid \theta_{0}, \phi_{0}\right)\right]$. To be useful for climate modeling, how this optical depth distribution depends on large-scale thermodynamic and dynamic variables (e.g., water vapor concentration, convective stability, and wind shear) would have to be known. Information about the $P\left(\tau \mid \theta_{0}, \phi_{0}\right)$ distribution could potentially be measured with instruments such as scanning microwave radiometers. A more likely approach for climate modeling would be to 1 ) express $P\left(\tau \mid \theta_{0}, \phi_{0}\right)$ in terms of relatively few parameters (e.g., by using gamma distributions in $\tau$ ), 2) run cloud dynamical models for a large number of forcing conditions, and 3 ) use the resulting model cloud fields to determine the parameters of the anisotropic optical depth distribution as a function of large-scale variables [e.g., see Hinkelman et al. (2005) for sample relations between vertical wind shear and anisotropy].

\section{c. Importance of anisotropy effects to various applications}

The results of this study indicate that not accounting for horizontal anisotropy or vertical tilt in cumulus cloud fields can cause errors of up to $40 \mathrm{~W} \mathrm{~m}^{-2}$ in instantaneous downwelling solar surface fluxes at spatial scales on the order of $10 \mathrm{~km}$. An important question is then whether anisotropy can significantly affect solar radiative parameters on longer temporal or larger spatial scales. In order for this to be the case, the following conditions would have to hold. First, the cloud fraction, optical depth, and aspect ratio of the cumulus clouds would need to be large enough for the field to produce significant 3D radiative effects regardless of the anisotropy. In addition, the anisotropic cloud field would have to exist over most of the period and region, the direction of the anisotropy would have to be uniform over the same intervals, and the relative orientation of the sun to the cloud features over the period of interest would need to not average out the relevant anisotropy effects. Based on the lifetime and extent of cumulus fields and midlatitude dynamic features, we might expect anisotropic fields to exist for at most 3-4 h over a region on the order of $100 \mathrm{~km}$. It is also conceivable that the type and direction of anisotropy in such fields would be reasonably uniform. Given this, the important question becomes how much any temporal and spatial averaging would reduce the anisotropy's radiative impact. Based on our results for random azimuthal angles, averaging would tend to minimize the effects of stretched clouds. However, for tilted clouds, substantial radiative effects could remain. This is because the effective cloud fraction increases with tilt for all solar azimuthal angles except $0^{\circ}$. Thus cumulus tilt could noticeably change solar transmission and albedo along with the diffuse ratio for scales as large as $100 \mathrm{~km}$ and a few hours. In cases with more extensive averaging in time or space, anisotropy effects are probably insignificant.

We can estimate the magnitude of the effect of anisotropy on transmitted solar flux for midlatitude continental fair-weather cumulus fields over a longer period based on the anisotropy values computed for such LES cumulus scenes in Hinkelman et al. (2005). Table 5 in that paper shows anisotropy values for LES runs using a variety of typical local environmental conditions vector averaged over $2.5 \mathrm{~h}$. The maximum anisotropy parameter value listed is 0.229 , which occurred for a vertical wind shear of $4 \mathrm{~m} \mathrm{~s}^{-1} \mathrm{~km}^{-1}$. Table 2 indicates a maximum change in the downward solar flux of about $5 \mathrm{~W} \mathrm{~m}^{-2}$ for a solar zenith angle of $60^{\circ}$ at this level of stretching. The maximum tilt listed is 0.731 , 
for the case with a uniform wind speed of $20 \mathrm{~m} \mathrm{~s}^{-1}$. This tilt could decrease the downward solar flux by as much as $13.4 \mathrm{~W} \mathrm{~m}^{-2}$, again for a $60^{\circ}$ solar zenith angle. Thus, anisotropy could produce $5-12 \mathrm{~W} \mathrm{~m}^{-2}$ variations in the downwelling surface solar flux. The importance of such variations would depend on the application.

Changes in atmospheric heating rate profiles due to tilt or horizontal anisotropy could alter the structure or microphysics of the clouds themselves. However, the impact of anisotropy on the development of cumulus clouds is difficult to estimate from the analysis presented here. The heating rates computed here, which vary less than $1 \mathrm{~K} \mathrm{day}^{-1}$ as cloud structure changes, are averages over the entire domain, including the clear areas. A 3D analysis of heating rate changes, which would identify "hot spots" (Vogelmann et al. 2001) within the clouds, would provide more insight into this matter. Large eddy simulations with interactive 3D radiative transfer could also shed light on this subject. Given that the wet adiabatic cooling rate is about $50 \mathrm{~K}$ $\mathrm{h}^{-1}$ (Cotton and Anthes 1989), anisotropy would have to cause pockets of very strong heating to have any impact on cloud development. It is more likely that anisotropy-induced solar heating would be effective after cloud development, tending to shorten cloud lifetimes. Further study is required to evaluate these effects.

It should be noted that the results described in this paper were obtained for a group of continental fairweather cumulus-type fields having similar properties and created by a stochastic model. All of the fields had comparable cloud fractions and aspect ratios, and cloud depths did not exceed $1.5 \mathrm{~km}$. However, an ensemble of 20 fields was used for each tilt or anisotropy state investigated. Our confidence in the reliability of the computed flux and heating rate trends for the type of cloud fields employed is therefore high. Nevertheless, the radiative effects of anisotropy and tilt in other types of cloud fields may differ. For example, we expect that cloud field distortions in cumulus with greater vertical development will produce larger changes in surface radiative fluxes while anisotropy effects for more overcast scenes are anticipated to be less significant.

As in most studies of radiative transfer in the cloudy atmosphere, certain simplifications were made in these computations. One such simplification was the use of a single average water vapor profile throughout the computational domain. It is true that saturating the cloudy cells would change the details of the computed heating rate profiles. However, tests (not shown) indicate that, although individual heating rate values may change by a few percent, using this alternative does not materially affect the trends as a function of anisotropy. Other as- sumptions included constant cloud droplet number density and distribution characteristics as well as the use of the McClatchey midlatitude summer atmospheric profile above $4300 \mathrm{~m}$. Despite any inaccuracy introduced by these simplifications, we expect the overall trends reported to hold since the greatest effect of anisotropy on radiative processes was due to gross changes in the apparent shape of cloud boundaries.

\section{Conclusions}

The radiative impact of horizontal elongation and vertical tilt of fair-weather cumulus clouds has been assessed. Statistics describing the structure of cumulus scenes produced by a large eddy simulation model were used as input to a stochastic field generation algorithm, which created a series of 20 LWC fields with similar characteristics. Incrementally greater $x-z$ plane tilt and horizontal stretching were imposed on each of the 20 fields, so that an ensemble of scenes was produced for each level of distortion. Broadband solar radiative transfer was then computed for each member of these ensembles using a Monte Carlo radiative transfer model. The domain-averaged solar fluxes, diffuse flux ratios, and atmospheric heating rate profiles for the scenes were calculated for several combinations of solar zenith and azimuth angles.

Both tilt and horizontal anisotropy (stretching) were found to significantly affect instantaneous domainaveraged solar fluxes, but tilt had more radiative impact than horizontal anisotropy. For example, the maximum change in the downward solar flux at the surface caused by tilt was $-39.9 \mathrm{~W} \mathrm{~m}^{-2}$; for stretch it was $-12.7 \mathrm{~W}$ $\mathrm{m}^{-2}$. Maximum changes relative to the corresponding mean isotropic downward solar flux were -0.072 for tilt and -0.032 for stretching. Changes in flux caused by cloud anisotropy strongly depended on both the solar zenith and azimuth angles in addition to the degree of anisotropy. For the stretched scenes, solar transmission increased when the direction of solar incidence was parallel to the stretching direction and decreased for broadside incidence. For the tilted scenes, transmission decreased for all solar azimuths except $0^{\circ}$ (i.e., when the clouds tilted toward the sun). At $0^{\circ}$, transmission increased as the cloud tilt approached the solar zenith angle. Changes in albedo were the opposite of the transmission changes. Although the magnitudes of the changes in the domain-averaged absorbed solar fluxes were small (maximum $5.0 \mathrm{~W} \mathrm{~m}^{-2}$ for tilt and $1.3 \mathrm{~W} \mathrm{~m}^{-2}$ for stretching), the spatial distortions of the cloud fields caused significant alterations (up to $0.65 \mathrm{~K} \mathrm{day}^{-1}$ for tilt and $0.25 \mathrm{~K} \mathrm{day}^{-1}$ for stretching) in the atmospheric heating rate profiles in many cases. 
Allowing the solar azimuth angle to vary randomly did not eliminate the effects of cumulus anisotropy, particularly vertical tilt. For random solar azimuths, the maximum change in the downward solar flux at the surface caused by tilt was $-27.5 \mathrm{~W} \mathrm{~m}^{-2}$ while for stretch it was $-3.5 \mathrm{~W} \mathrm{~m}^{-2}$. For scenes with little tilt or horizontal anisotropy, these values were much smaller $\left(-1\right.$ to $\left.-5 \mathrm{~W} \mathrm{~m}^{-2}\right)$. It should be noted, though, that applying completely random azimuths is an extreme situation, since the solar azimuth varies over a limited range even over extended periods at most locations.

Independent pixel and tilted independent pixel approximation (IPA and TIPA, respectively) radiative transfer calculations were also performed for all of the stochastic scenes. Comparison of domain-averaged solar quantities computed using the independent pixel approximation and the full three-dimensional Monte Carlo model showed that the IPA was unable to achieve reasonable accuracy for the tilted and stretched cumulus fields at most angles of solar incidence. This was because the bulk of the radiative effect of the distorted cloud fields was due to changes in their effective cloud fraction. (By definition, the IPA is incapable of modeling the incidence of radiation on cloud sides and so is only sensitive to nadir cloud fraction.) Errors in the TIPA flux results were more predictable: always negative and on the order of $10 \mathrm{~W} \mathrm{~m}^{-2}$. The uniformity of TIPA flux errors implies that photon transport perpendicular to the direction of incidence depends only on basic cloud structure and properties. This makes TIPA more useful than IPA for approximate calculations of radiative transfer through anisotropic clouds. A possible parameterization approach for the inclusion of anisotropy in TIPA computations when the full 3D structure of the cloud field is unknown was outlined.

Changes in solar radiative propagation due to cumulus anisotropy may be important in some situations. While anisotropy effects can be reduced by the relative motion of the sun over time or the variability of cloud shapes throughout a region, we estimate that cumulus anisotropy could produce $5-12 \mathrm{~W} \mathrm{~m}^{-2}$ variations in the downwelling solar surface flux over periods of $\sim 2.5 \mathrm{~h}$ under the most conducive circumstances. Substantially larger variations can occur on shorter temporal scales. Nevertheless, the tilted independent pixel approximation has been found to handle the radiative effects of cloud anisotropy well. Unless extremely accurate results are required, use of TIPA to treat solar radiative transfer through anisotropic cumulus fields should be sufficient for most applications.

Acknowledgments. The authors thank Jason Cole, Chuck Pavloski, and Douglas Mast for helpful discus- sions and advice during the conduct of this research. The majority of this project was carried out while the first author was a graduate student at the Pennsylvania State University, supported in part under a National Science Foundation Graduate Fellowship. Additional funding was provided by the Environmental Sciences Division of the U.S. Department of Energy (Grants DE-FG02-90ER-61071 and DE-A1005-90ER-61069) as part of the Atmospheric Radiation Measurement Program, Battelle, Inc. (Contracts 354048-AQ5 and 354879-AQ5), and NASA's Science Mission Directorate through the GEWEX SRB and CERES projects.

\section{REFERENCES}

Aida, M., 1977: Reflection of solar radiation from an array of cumuli. J. Meteor. Soc. Japan, 55, 174-181.

Barker, H. W., 1994: Solar radiative transfer for wind-sheared cumulus cloud fields. J. Atmos. Sci., 51, 1141-1156.

_ 1996: Estimating cloud field albedo using one-dimensional series of optical depth. J. Atmos. Sci., 53, 2826-2837.

— scale-invariant broken cloud fields. J. Atmos. Sci., 49, 11151126.

— J.-J. Morcrette, and G. D. Alexander, 1998: Broadband solar fluxes and heating rates for atmospheres with 3D broken clouds. Quart. J. Roy. Meteor. Soc., 124, 1245-1271.

_ , and Coauthors, 2003: Assessing 1D atmospheric solar radiative transfer models: Interpretation and handling of unresolved clouds. J. Climate, 16, 2676-2699.

Benner, T. C., and K. F. Evans, 2001: Three-dimensional solar radiative transfer in small tropical cumulus fields derived from high-resolution imagery. J. Geophys. Res., 106, 14975 14984.

Cahalan, R. F., 1989: Overview of fractal clouds. RSRM '87: Advances in Remote Sensing Retrieval Methods, A. Deepak et al., Eds., A. Deepak Publishing, 371-388.

—, W. Ridgway, W. J. Wiscombe, T. L. Bell, and J. B. Snider, 1994a: The albedo of fractal stratocumulus clouds. J. Atmos. Sci., 51, 2434-2455.

,,,--- S. Gollmer, and Harshvardhan, 1994b: Independent pixel and Monte Carlo estimates of stratocumulus albedo. J. Atmos. Sci., 51, 3776-3790.

Chambers, L. H., B. A. Wielicki, and K. F. Evans, 1997: Independent pixel and two-dimensional estimates of Landsat-derived cloud field albedo. J. Atmos. Sci., 54, 1525-1532.

Cotton, W. R., and R. A. Anthes, 1989: Storm and Cloud Dynamics. International Geophysical Series, Vol. 44, Academic Press, 883 pp.

Davies, R., 1978: The effect of finite geometry on the threedimensional transfer of solar irradiance in clouds. J. Atmos. Sci., 35, 1712-1725.

Di Giuseppe, F., and A. M. Tompkins, 2005: Impact of cloud cover on solar radiative biases in deep convective regimes. $J$. Atmos. Sci., 62, 1989-2000.

Evans, K. F., 1993: Two-dimensional radiative transfer in cloudy atmospheres: The spherical harmonic spatial grid method. $J$. Atmos. Sci., 50, 3111-3124.

— , and W. J. Wiscombe, 2004: An algorithm for generating stochastic cloud fields from radar profile statistics. Atmos. Res., 72, 263-289. 
Harshvardhan, and R. W. L. Thomas, 1984: Solar reflection from interacting and shadowing cloud elements. J. Geophys. Res., 89, 7179-7185.

Hinkelman, L. M., B. Stevens, and K. F. Evans, 2005: A largeeddy simulation study of anisotropy in fair-weather cumulus cloud fields. J. Atmos. Sci., 62, 2155-2171.

Marchuk, G. I., G. A. Mikhailov, M. A. Nazaraliev, R. A. Darbinjan, B. A. Kargin, and B. S. Elepov, 1980: The Monte Carlo Methods in Atmospheric Optics. Springer Series in Optical Sciences, Vol. 12, Springer-Verlag, 208 pp.

Marshak, A., A. Davis, W. Wiscombe, and R. Cahalan, 1995a: Radiative smoothing in fractal clouds. J. Geophys. Res., 100, $26247-26261$.

,--1, and G. Titov, 1995b: The verisimilitude of the independent pixel approximation used in cloud remote sensing. Remote Sens. Environ., 52, 71-78.

McKee, T. B., and S. K. Cox, 1974: Scattering of visible radiation by finite clouds. J. Atmos. Sci., 31, 1885-1892.

Miles, N. L., J. Verlinde, and E. E. Clothiaux, 2000: Cloud droplet size distributions in low-level stratiform clouds. J. Atmos. Sci., 57, 295-311.

Mlawer, E. J., S. J. Taubman, P. D. Brown, M. J. Iacono, and S. A. Clough, 1997: Radiative transfer for inhomogeneous atmospheres: RRTM, a validated correlated-k model for the longwave. J. Geophys. Res., 102, 16 663-16 682.
O'Hirok, W., and C. Gautier, 1998: A three-dimensional radiative transfer model to investigate the solar radiation within a cloudy atmosphere. Part I: Spatial effects. J. Atmos. Sci., 55, 2162-2179.

Ronnholm, K., M. B. Baker, and H. Harrison, 1980: Radiative transfer through media with uncertain or variable parameters. J. Atmos. Sci., 37, 1279-1290.

Scheirer, R., and A. Macke, 2003: Cloud inhomogeneity and broadband solar fluxes. J. Geophys. Res., 108, 4599, doi:10.1029/2002JD003321.

Titov, G. A., 1998: Radiative horizontal transport and absorption in stratocumulus clouds. J. Atmos. Sci., 55, 2549-2560.

Várnai, T., and R. Davies, 1999: Effects of cloud heterogeneities on shortwave radiation: Comparison of cloud top variability and internal heterogeneity. J. Atmos. Sci., 56, 4206-4224.

Vogelmann, A. M., V. Ramanathan, and I. A. Podgorny, 2001: Scale dependence of solar heating rates in convective cloud systems with implications to general circulation models. $J$. Climate, 14, 1738-1752.

Welch, R. M., and B. A. Wielicki, 1984: Stratocumulus cloud field reflected fluxes: The effect of cloud shape. J. Atmos. Sci., 41, 3085-3103.

Zuidema, P., and K. F. Evans, 1998: On the validity of the independent pixel approximation for boundary layer clouds observed during ASTEX. J. Geophys. Res., 103, 6059-6074. 